\title{
New concepts of inverse fuzzy mixed graphs and its application
}

\author{
Soumitra Poulik ${ }^{1} \cdot$ Ganesh Ghorai ${ }^{1}$ (D)
}

Received: 6 June 2021 / Accepted: 22 August 2021 / Published online: 6 September 2021

(C) The Author(s), under exclusive licence to Springer Nature Switzerland AG 2021

\begin{abstract}
Fuzzy mixed graph (FMG) can be used to model some graphical intercommunication network systems if there are many directed and undirected relations between some vertices. In inverse fuzzy graph, the membership value of edges are greater than or equal to the minimum of the membership value of the corresponding vertices. In inverse fuzzy mixed graph (IFMG), directed and undirected relations exist between some vertices and it can be used to analyze many graphical problems of real life such that the membership values of edges are greater than or equal to the minimum of the membership value of the corresponding pair of vertices. In this article, the concept of IFMG is introduced first with some of its properties. Then some isomorphic properties are studied and complement of IFMG is given. Different types of operations like union, intersection, product and join between two IFMGs are defined and investigated some of their related results. An algorithm of the proposed method is executed to identify some vertices. An application is depicted using the concept IFMG to examine the order of vertices in a social network group according to communication gaps.
\end{abstract}

Keywords Inverse fuzzy mixed graph · Complement · Isomorphism · Union · Intersection and Join

\section{Introduction}

In 1965, Zadeh (1965) first introduced the uncertainty concepts in mathematics which is known as fuzzy sets. Graph theory has many applications in various connected fields like network routing, internet path systems, computer science and many shortest path problems. Li et al. (2020, 2021) introduced Elephant herding optimization in variants, hybrids and a Survey of based on intelligent optimization with their real applications and algorithms in different environments. Feng et al. (2021) introduced Monarch butterfly optimization (MBO) algorithm, hybridizations, variants and applications in optimization techniques to solve global numerical optimization and engineering problems. Wang et al. (2019) gave comprehensive review of different $\mathrm{KH}$ algorithms and analyzed

Ganesh Ghorai

math.ganesh@mail.vidyasagar.ac.in

Soumitra Poulik

poulikmsoumitra@gmail.com

1 Department of Applied Mathematics with Oceanology and Computer Programming, Vidyasagar University, Midnapore 721102, India their applications in engineering and optimization with some future directions.

In 1975, Rosenfeld (1975) first used the concept of fuzzy logic in graph theory and introduced concept fuzzy graph. Recently, concept of fuzzy graphs have been used in many uncertain connected fields including signal processing, image segmentation, computer science, medical and engineering science, water and electric network systems. Mordeson and Peng (1994) defined different types of operation on fuzzy graphs likes union, intersection and join of two fuzzy graphs. Different types strong arc, weak arc between two vertices in fuzzy graph are discussed by Mathew and Sunitha (2009). Mathew et al. (2018a, b) introduced many indices, path and connectivity in fuzzy graph with application to human trafficking. Das et al. (2021) introduced fuzzy chordal graphs and their properties. Bhutani and Rosenfeld (2003) introduced end nodes and their character in various fuzzy graphs. Binu et al. $(2019,2020)$ established connectivity and wiener index in fuzzy graphs and explained their property with some real applications like illegal immigration, human trafficking between countries. Poulik and Ghorai (2020, 2021) introduced many indices in bipolar fuzzy graphs and explained their properties with applications in different types of journeys order and social development. Some boundary 
and interior nodes of bipolar fuzzy graphs and their application in education systems are explained by Poulik and Ghorai (2020a, b). Turksen (1986) introduced interval valued fuzzy sets based on normal forms. Then, Chen (1997a); Chen et al. (1997); Chen and Hsiao (2000) introduced different types of bidirectional ruled based reasoning and applications of interval-valued fuzzy hypergraphs. Mendel et al. (2006) introduced intervalvalued type-2 fuzzy logic. Then, Chen et al. $(2012,2013)$ introduced different types of interval-valued type-2 fuzzy sets and their applications in decision making. Knyazeva et al. (2018) introduced topological ordering on intervalvalued type-2 fuzzy graphs. Singh $(2018,2019,2020)$ introduced different types of lattices with $m$-polar fuzzy concepts and neutrosophic concepts and their applications. Yager $(2020,2021)$ introduced many mathematical models in uncertain environment and their applications.

One of the main role of vertices and edges in a fuzzy graph depend on the total membership values of vertices and edges. Cary (2018) and Gani and Ahamed (2003) explained the order and size of fuzzy graphs and the regularity of vertices and edges. Ghorai and Pal (2016) and Ghorai and Jacob (2019) introduced degree of vertices in different types of fuzzy graphs. Some important algorithms and domination on fuzzy graph are discussed by West (2000) and Mariappan et al. (2019). Kumar and Lavanya (2017) and Nirmala and Prabavathi (2015) explained fuzzy diagraphs and their mathematical tools with application. Rashmanlou et al. (2020) introduced novel application of cubic graph based on fuzzy concept. Samanta and Pal (2020), Samanta et al. (2021, 2021) introduced measure of the influence in social networks, fuzzy planar graphs and different directed graphs with their application in social media and image processing. Akram $(2011,2013)$ and Akram and Waseem (2018) described different types of bipolar fuzzy graphs with order, size, isomorphic properties and some real applications. Some properties of bipolar and $m$-polar fuzzy graph and their union, join, complement, etc are examined by Akram (2018) and Akram et al. (2020). Akram and Shahzadi (2021) introduced concepts of Pythagorean dombi fuzzy soft graphs. They also have discussed some decision making problems by using Pythagorean dombi fuzzy soft graphs. Akram and Dudek (2010) introduced interval-valued fuzzy graphs. Borozooei et al. (2020) introduced the concepts of inverse of fuzzy graphs and their properties. Das et al. (2021) introduced fuzzy mixed graph with application in COVID-19 affected central regions.

In many graphical network systems, the vertices may not be always active. So the activation time and the inactivation time are uncertain. Therefore, the disconnection time between vertices are also uncertain. Again, the disconnection time between a pair of vertices is greater than or equal to minimum of the inactivation times of those pair of vertices. These types problems can be solved by using the concept of inverse fuzzy graphs. If in a fuzzy graphical system there exists some directed relationship between vertices, then the FMG can be used to model the system. In many real world problems with inverse fuzzy graph structure, there may exist directed relationship between vertices. This type of situations can not be modeled well by means of our familiar graphs. So this motivates us to define IFMG which can handle the above situations.

This paper is structured as follows: the next Section contains some basic definitions of different types of fuzzy graphs. In Sect. 3, IFMG is defined and some important theorems are explained by examples. Some operations like intersection, union and Cartesian product on two IFMGs and some theorems related to these operations are described in Sect. 5. An algorithm to calculate the degree of vertices and to find the important vertices in IFMG is given in Sect. 5. In Sect. 6, we construct a network of some friends and then by using the concept of IFMG the order of the friends for communication gap are discussed. At last conclusion with some future works of this work is depicted in Sect. 7.

\section{Preliminaries}

Here some basic definitions are given. The bold notations like ab denote the directed edges in the whole paper.

Definition 1 (Rosenfeld 1975) A fuzzy graph $\zeta=$ $(V, \mu, \rho)$ is a non-empty set $\mathrm{V}$ together with a pair of functions $\mu: V \rightarrow[0,1]$ and $\rho: V \times V \rightarrow[0,1]$ such that for all $x, y \in V, \rho(x y) \leq \mu(x) \wedge \mu(y)$ where $\mu(x)$ and $\rho(x y)$ represent the membership values of the vertex $x$ and of the edge $x y$ in $\zeta$ respectively.

Definition 2 (Mordeson and Peng 1994) A sequence of distinct vertices $x_{0}, x_{1}, \ldots, x_{n}$ is called a path $P$ of length $n$ if $\rho\left(a_{i-1}, a_{i}\right)>0, i=1,2, \ldots, n$. The membership value of the weakest edges is called the strength of the path $P$. Two FGs $\zeta=(\mu, \rho)$ and $\zeta^{\prime}=\left(\mu^{\prime}, \rho^{\prime}\right)$ are isomorphic to each other if there exists a bijective mapping $f$ from $\zeta$ to $\zeta^{\prime}$ such that $\mu\left(x_{i}\right)=\mu^{\prime}\left(f\left(x_{i}\right)\right), \forall x_{i} \in \mu$ and $\rho\left(x_{i} x_{j}\right)=$ $\rho^{\prime}\left(f\left(x_{i} x_{j}\right)\right), \forall x_{i} x_{j} \in \rho^{*}$.

Definition 3 (Borozooei et al. 2020) Let $\zeta^{*}=(V, E)$ be a simple graph and $\mu: V \rightarrow[0,1]$ and $\rho: E \rightarrow[0,1]$ be two fuzzy subsets of $V$ and $E$ respectively. Then $\zeta=(\mu, \rho)$ is said to be an inverse fuzzy graph if for any $x y \in E$, $\rho(x y) \geq \min \{\mu(x), \mu(y)\}$.

Definition 4 (Borozooei et al. 2020) Let $\zeta=(\mu, \rho)$ be an inverse fuzzy graph. Then the degree of a vertex $x \in V$ is 
defined by $d(x)=\sum_{x y \in E} \rho(x y)$. The order of $\zeta$ is $\sum_{x \in V} \mu(x)$ and the size of $\zeta$ is $\sum_{x y \in E} \rho(x y)$.

Definition 5 (Das et al. 2021) Let $V$ be a non empty set and $E_{1}, E_{2} \subseteq V \times V$. Then $\zeta=\left(V, E_{1}, E_{2}, \rho_{1}, \rho_{2}, \mu, \tau\right)$ is called an IFMG, where $\mu: V \rightarrow[0,1], \rho_{1}: E_{1} \rightarrow[0,1]$ and $\rho_{2}, \tau: E_{2} \rightarrow[0,1]$ such that $\rho_{1}(x y) \leq \mu(x) \wedge \mu(y), \forall x y \in$ $E_{1}, \quad \rho_{2}(\mathbf{x y}) \leq \mu(x) \wedge \mu(y), \forall \mathbf{x y} \in E_{2}$, and $\tau(\mathbf{x y}) \leq \mid \mu(x)-$ $\mu(y) \mid, \forall \mathbf{x y} \in E_{2}$. Here $\mu$ is the membership values of vertices, $\rho_{1}$ and $\rho_{2}$ represents the membership values of undirected and directed edges respectively.

Definition 6 (Das et al. 2021) Let $\zeta=$ $\left(V, E_{1}, E_{2}, \sigma_{1}, \sigma_{2}, \mu, \tau\right)$ be a FMG. Then the order of $\zeta$ is $\sum_{x \in V} \mu(x)$ and the size of $\zeta$ is

$$
\left(\sum_{x y \in E_{1}} \sigma_{1}(x y), \sum_{\mathbf{x y} \in E_{2}} \sigma_{1}(\mathbf{x y}), \sum_{\mathbf{x y} \in E_{2}} \tau(\mathbf{x y})\right) .
$$

\section{Inverse fuzzy mixed graph (IFMG)}

In this section, first the definition of IFMG is given and then the complement of an IFMG is defined. Some isomorphic properties of it are also established.

Definition 7 Let $V$ be a non-void set and $E_{1}, E_{2} \subseteq V \times V$. Then $\zeta=\left(V, E_{1}, E_{2}, \sigma_{1}, \sigma_{2}, \mu, \tau\right)$ is called an IFMG, where $\mu: V \rightarrow[0,1], \sigma_{1}: E_{1} \rightarrow[0,1]$ and $\sigma_{2}, \tau: E_{2} \rightarrow[0,1]$ such that

$\sigma_{1}(a b) \geq \mu(a) \wedge \mu(b), \forall a b \in E_{1}$,

$\sigma_{2}(\mathbf{a b}) \geq \mu(a) \wedge \mu(b), \forall \mathbf{a b} \in E_{2}$,

and

$\tau(\mathbf{a b}) \geq|\mu(a)-\mu(b)|, \forall \mathbf{a b} \in E_{2}$.

Here $\mu$ represents the membership value of vertices, $\sigma_{1}$ and $\sigma_{2}$ represent the membership value of undirected and directed edges respectively and $\tau$ denote the measure of directedness for undirected edges.

Example 1 In Fig. 1, we consider $V=\{a, b, c, d\}, \mu(a)=$ $0.4, \mu(b)=0.3, \mu(c)=0.3, \quad \mu(d)=0.2 \quad$ and $\quad E_{1}=$ $\{a b, a c, a d, b d, c d\}, \sigma_{1}(a b)=0.4, \sigma_{1}(a c)=0.3, \sigma_{1} \quad(a d)$ $=0.3, \sigma_{1}(b d)=0.4, \sigma_{1}(c d)=0.2$. So, $\sigma_{1}(a b) \geq \mu(a) \wedge$ $\mu(b), \sigma_{1}(a c) \geq \mu(a) \wedge \mu(c), \sigma_{1}(a d) \geq \mu(a) \quad \wedge \mu(d), \sigma_{1}(b d)$ $\geq \mu(b) \wedge \mu(d), \sigma_{1}(c d) \geq \mu(c) \wedge \mu(d)$.

Also let $E_{2}=\{\mathbf{a b}, \mathbf{c d}, \mathbf{d c}, \mathbf{b d}\}$ and $\sigma_{2}(\mathbf{a b})=0.3, \sigma_{2}(\mathbf{c d})$ $=0.3, \sigma_{2}(\mathbf{d c})=0.2, \sigma_{2}(\mathbf{b d})=0.3$.

Therefore, $\sigma_{2}(\mathbf{a b}) \geq \mu(a) \wedge \mu(b), \sigma_{2}(\mathbf{c d}) \geq \mu(c) \wedge \mu(d)$, $\sigma_{2}(\mathbf{d c}) \geq \mu(d) \wedge \mu(c), \sigma_{2}(\mathbf{b d}) \geq \mu(b) \wedge \mu(d)$.

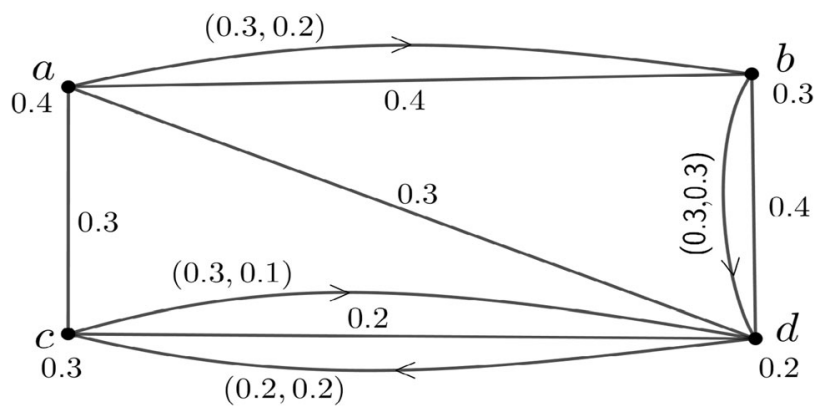

Fig. 1 An IFMG $\zeta$

$\tau(\mathbf{a b})=0.2,|\mu(a)-\mu(b)|=0.1$. So, $\quad \tau(\mathbf{a b}) \geq \mid \mu(a)-$ $\mu(b) \mid$.

Similarly, $\quad \tau(\mathbf{c d}) \geq|\mu(c)-\mu(d)|, \tau(\mathbf{d c}) \geq|\mu(d)-\mu(c)|$ ,$\tau(\mathbf{b d}) \geq|\mu(b)-\mu(d)|$.

Hence $\zeta=\left(V, E_{1}, E_{2}, \sigma_{1}, \sigma_{2}, \mu, \tau\right)$ is an IFMG.

Definition 8 Let $\zeta=\left(V, E_{1}, E_{2}, \sigma_{1}, \sigma_{2}, \mu, \tau\right)$ be an IFMG and $V=V^{c}, E_{1}^{c}, E_{2}^{c} \subseteq V \times V$. The complement of $\zeta$ is denoted by $\zeta^{c}$ and is defined as $\zeta^{c}=\left(V, E_{1}^{c}\right.$, $\left.E_{2}^{c}, \sigma_{1}^{c}, \sigma_{2}^{c}, \mu^{c}, \tau^{c}\right) \quad$ where $\mu^{c}: V \rightarrow[0,1], \sigma_{1}^{c}: E_{1}^{c} \rightarrow[0,1]$ and $\sigma_{2}^{c}, \tau^{c}: E_{2}^{c} \rightarrow[0,1]$ such that

$\mu^{c}(a)=\mu(a), \forall a \in V$,

$\sigma_{1}^{c}(a b)=1-\sigma_{1}(a b)+\mu(a) \wedge \mu(b), \forall a b \in E_{1}^{c}$,

$\sigma_{2}^{c}(\mathbf{a b})=1-\sigma_{2}(\mathbf{a b})+\mu(a) \wedge \mu(b), \forall \mathbf{a b} \in E_{2}^{c}$,

and

$\tau^{c}(\mathbf{a b})=1-\tau(\mathbf{a b})+|\mu(a)-\mu(b)|, \forall \mathbf{a b} \in E_{2}^{c}$.

Example 2 In Fig. 2, we consider $V=V^{c}=$ $\{a, b, c, d\}, \mu(a)=\mu^{c}(a)=0.4, \mu(b)=\mu^{c}(b)=0.3, \mu(c)$ $=\mu^{c}(c)=0.3, \mu(d)=\mu^{c}(d)=0.2$ and $E_{1}^{c}=\{a b, a c, a d$, $b d, c d\}, E_{2}^{c}=\{\mathbf{a b}, \mathbf{c d}, \mathbf{d c}, \mathbf{b d}\}$. Now, from Example 1, we have $1-\sigma_{1}(a b)+\mu(a) \wedge \mu(b)=1-0.4+0.3=0.9=$ $\sigma_{1}^{c}(a b)$.

Similarly, $\quad \sigma_{1}^{c}(a c)=1-\sigma_{1}(a c)+\mu(a) \wedge \mu(c)=1, \sigma_{1}^{c}$ $(a d)=1-\sigma_{1}(a d)+\mu(a) \wedge \mu(d)=0.9, \quad \sigma_{1}^{c}(b d)=1-\sigma_{1}$ $(b d)+\mu(b) \wedge \mu(d)=0.8, \sigma_{1}^{c}(c d)=1-\sigma_{1}(c d)+\mu(c) \wedge$ $\mu(d)=1$. So, $\sigma_{1}^{c}(a b) \geq \mu^{c}(a) \wedge \mu^{c}(b), \forall a b \in E_{1}^{c}$.

$E_{2}^{c}=\{\mathbf{a b}, \mathbf{c d}, \mathbf{d c}, \mathbf{b d}\} .1-\sigma_{2}(\mathbf{a b})+\mu(a) \wedge \mu(b)=1-$ $0.3+0.3=1=\sigma_{2}^{c}(\mathbf{a b})$.

Similarly, $\sigma_{2}^{c}(\mathbf{c d})=1-\sigma_{2}(\mathbf{c d})+\mu(c) \wedge \mu(d)=0.9, \sigma_{2}^{c}$ $(\mathbf{d c})=1-\sigma_{2}(\mathbf{d c})+\mu(d) \wedge \mu(c)=1, \sigma_{2}^{c}(\mathbf{b d})=1-\sigma_{2}$ $($ bd $)+\mu(b) \wedge \mu(d)=0.9$. So, $\quad \sigma_{2}^{c}(\mathbf{a b}) \geq \mu^{c}(a) \wedge \mu^{c}(b)$, $\forall \mathbf{a b} \in E_{2}^{c}$.

$1-\tau(\mathbf{a b})+|\mu(a)-\mu(b)|=\quad 1-0.2+0.1=0.9=$ $\tau^{c}(\mathbf{a b})$. 


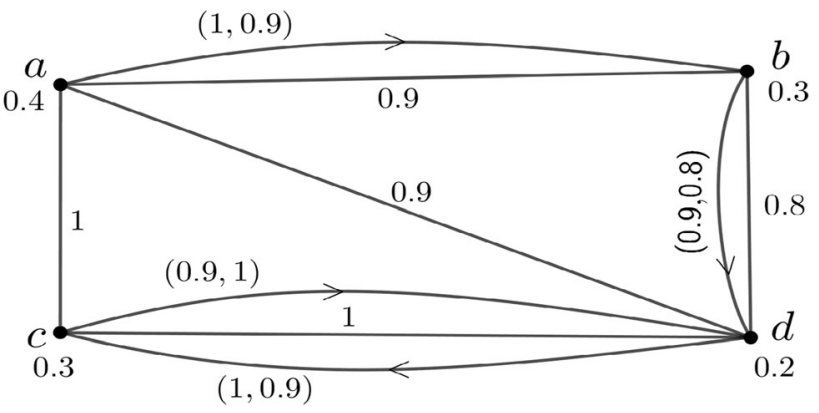

Fig. $2 \zeta^{c}$, the complement of IFMG $\zeta$ of Fig. 1

Similarly, $\quad \tau^{c}(\mathbf{c d})=1-\tau(\mathbf{c d})+|\mu(c)-\mu(d)|=1$, $\tau^{c}(\mathbf{d c})=1-\tau(\mathbf{d c})+|\mu(d)-\mu(c)|=0.9, \tau^{c}(\mathbf{b d})=1-$ $\tau(\mathbf{b d})+|\mu(b)-\mu(d)|=0.8$. So, $\tau^{c}(\mathbf{a b}) \geq\left|\mu^{c}(a)-\mu^{c}(b)\right|$, $\forall \mathbf{a b} \in E_{2}^{c}$.

Therefore, $\zeta^{c}=\left(V^{c}, E_{1}^{c}, E_{2}^{c}, \sigma_{1}^{c}, \sigma_{2}^{c}, \mu^{c}, \tau^{c}\right)$ is the complement of the IFMG $\zeta$ of Fig. 1. Also, $\zeta^{c}$ satisfies all the conditions of IFMG. Hence $\zeta^{c}$ is an IFMG.

Theorem 1 Let $\zeta=\left(V, E_{1}, E_{2}, \sigma_{1}, \sigma_{2}, \mu, \tau\right)$ be an IFMG. Then $\zeta^{c}=\left(V, E_{1}^{c}, E_{2}^{c}, \sigma_{1}^{c}, \sigma_{2}^{c}, \mu^{c}, \tau^{c}\right)$ is an IFMG and $\left(\zeta^{c}\right)^{c}=\zeta$.

Proof Consider the IFMG $\zeta$ and its complement $\zeta^{c}$. Then $\mu^{c}(a)=\mu(a), \forall a \in V$.

$\sigma_{1}(a b) \geq \mu(a) \wedge \mu(b) \Rightarrow \sigma_{1}(a b)-\mu(a) \wedge \mu(b)$

$\geq 0 \Rightarrow 1-\left(\sigma_{1}(a b)-\mu(a) \wedge \mu(b)\right) \leq 1$

$\Rightarrow \sigma_{1}^{c}(a b) \leq 1, \forall a b \in E_{1}^{c}$.

$\sigma_{1}(a b) \leq 1 \Rightarrow 1-\sigma_{1}(a b) \geq 0 . \quad \mu(a) \wedge \mu(b) \geq 0 \Rightarrow$

$1-\sigma_{1}(a b)+\mu(a) \wedge \mu(b) \geq \mu(a) \wedge \mu(b)$

$\Rightarrow \sigma_{1}^{c}(a b) \geq \mu(a) \wedge \mu(b)$.

Therefore $0 \leq \sigma_{1}^{c}(a b) \leq 1 \quad$ and $\quad \sigma_{1}^{c}(a b) \geq \mu(a) \wedge \mu(b)$, $\forall a b \in E_{1}^{c}$.

Similarly, $\quad 0 \leq \sigma_{2}^{c}(\mathbf{a b}) \leq 1 \quad$ and $\quad \sigma_{2}^{c}(\mathbf{a b}) \geq \mu(a) \wedge \mu(b)$, $\forall \mathbf{a b} \in E_{2}^{c}$.

$$
\begin{gathered}
\tau(\mathbf{a b}) \geq|\mu(a)-\mu(b)| \Rightarrow \tau(\mathbf{a b})-|\mu(a)-\mu(b)| \geq 0 \\
\Rightarrow 1-(\tau(\mathbf{a b})-|\mu(a)-\mu(b)|) \leq 1 \Rightarrow \tau^{c}(\mathbf{a b}) \leq 1, \forall \mathbf{a b} \in E_{2}^{c} .
\end{gathered}
$$

$\tau(\mathbf{a b}) \leq 1 \Rightarrow 1-\tau(\mathbf{a b}) \geq 0$. Since $|\mu(a)-\mu(b)| \geq 0$, so

$1-\tau(\mathbf{a b})+|\mu(a)-\mu(b)| \geq|\mu(a)-\mu(b)|$

$\Rightarrow \tau^{c}(\mathbf{a b}) \geq|\mu(a)-\mu(b)|$.

Therefore $\quad 0 \leq \tau_{2}^{c}(\mathbf{a b}) \leq 1 \quad$ and $\quad \tau_{2}^{c}(\mathbf{a b}) \geq \mid \mu(a)$ $-\mu(b) \mid, \forall \mathbf{a b} \in E_{2}^{c}$.

Now, $\zeta^{c}=\left(V, E_{1}^{c}, E_{2}^{c}, \sigma_{1}^{c}, \sigma_{2}^{c}, \mu^{c}, \tau^{c}\right)$ satisfies all the conditions of an IFMG.

Hence, $\zeta^{c}$ is an IFMG.

Again, $\left(V^{c}\right)^{c}=V$ and $\left(\mu^{c}\right)^{c}(a)=\mu^{c}(a)=\mu(a), \forall a \in V$. $\left(\sigma_{1}^{c}\right)^{c}(a b)=1-\sigma_{1}^{c}(a b)+\mu(a) \wedge \mu(b) \quad=1-(1-$ $\left.\sigma_{1}(a b)+\mu(a) \wedge \mu(b)\right)+\mu(a) \wedge \mu(b)=\sigma_{1}(a b), \forall a b \in E_{1}^{c}$. $\left(\sigma_{2}^{c}\right)^{c}(\mathbf{a b})=\quad 1-\sigma_{2}^{c}(\mathbf{a b})+\mu(a) \wedge \mu(b)=1-(1-$ $\left.\sigma_{2}(\mathbf{a b})+\mu(a) \wedge \mu(b)\right)+\mu(a) \wedge \mu(b)=\sigma_{2}(\mathbf{a b}), \forall \mathbf{a b} \in E_{2}^{c}$.

$\left(\tau^{c}\right)^{c}(\mathbf{a b})=1-\tau^{c}(\mathbf{a b})+|\mu(a)-\mu(b)|=1-(1-$ $\tau(\mathbf{a b})+|\mu(a)-\mu(b)|)+\mu(a) \wedge \mu(b)=\tau(\mathbf{a b}), \forall \mathbf{a b} \in E_{2}^{c}$.

Therefore, $\left(E_{1}^{c}\right)^{c}=E_{1}$ and $\left(E_{1}^{c}\right)^{c}=E_{1}$. Hence, $\left(\zeta^{c}\right)^{c}=\zeta$.

Example 3 Consider the IFMGs $\zeta$ and $\zeta^{c}$ of Examples 1 and 2. Here, $\left(\mu^{c}\right)^{c}(a)=\mu^{c}(a)=\mu(a), \forall a \in V$ obviously. Now, $\quad\left(\sigma_{2}^{c}\right)^{c}(\mathbf{a b})=1-\sigma_{2}^{c}(\mathbf{a b})+\mu(a) \wedge \mu(b)=1-0.9+$ $0.3=0.4=\sigma_{1}(a b) . \quad$ Similarly, $\left(\sigma_{1}^{c}\right)^{c}(a c)=\sigma_{1}(a c)$, $\left(\sigma_{1}^{c}\right)^{c}(a d)=\sigma_{1}(a d),\left(\sigma_{1}^{c}\right)^{c}(b d)=\sigma_{1}(b d),\left(\sigma_{1}^{c}\right)^{c}(c d)=$ $\sigma_{1}(c d)$.

$\left(\sigma_{2}^{c}\right)^{c}(\mathbf{a b})=1-\sigma_{2}^{c}(\mathbf{a b})+\mu(a) \wedge \mu(b)=1-1+0.3=$ $0.3=\sigma_{2}(\mathbf{a b})$. Similarly, $\left(\sigma_{2}^{c}\right)^{c}(\mathbf{c d})=\sigma_{2}(\mathbf{c d}),\left(\sigma_{2}^{c}\right)^{c}(\mathbf{d c})=$ $\sigma_{2}(\mathbf{d c}),\left(\sigma_{2}^{c}\right)^{c}(\mathbf{b d})=\sigma_{2}(\mathbf{b d})$.

$\left(\tau^{c}\right)^{c}(\mathbf{a b})=1-\tau^{c}(\mathbf{a b})+|\mu(a)-\mu(b)|=1-0.9$ $+0.1=0.2=\tau(\mathbf{a b})$. Similarly,

$$
\left(\tau^{c}\right)^{c}(\mathbf{c d})=\tau(\mathbf{c d}),\left(\tau^{c}\right)^{c}(\mathbf{d c})=\tau(\mathbf{d c}),\left(\tau^{c}\right)^{c}(\mathbf{b d})=\tau(\mathbf{b d})
$$

. Hence, $\left(\zeta^{c}\right)^{c}=\zeta$.

Definition 9 Let $\zeta=\left(V, E_{1}, E_{2}, \sigma_{1}, \sigma_{2}, \mu, \tau\right)$ be an IFMG. Then the order of $\zeta$ is $o(\zeta)=\sum_{a \in V} \mu(a)$ and the size of $\zeta$ is $S(\zeta)=\left(s_{1}, s_{2}, s_{3}\right)$, where $s_{1}=\sum_{a b \in E_{1}} \sigma_{1}(a b)$,

$$
s_{2}=\sum_{\mathbf{a b} \in E_{2}} \sigma_{1}(\mathbf{a b}) \text { and } s_{3}=\sum_{\mathbf{a b} \in E_{2}} \tau(\mathbf{a b}) \text {. }
$$

Example 4 Consider the IFMGs $\zeta$ of Example 1. Here $o(\zeta)=\mu(a)+\mu(b)+\mu(c)+\mu(d)=0.4+0.3+0.3+$ $0.2=1.2 . \quad s_{1}=\sigma_{1}(a b)+\sigma_{1}(a c)+\sigma_{1}(a d)+\sigma_{1}(b d)+$ $\sigma_{1}(c d)=0.4 \quad+0.3+0.3+0.4+0.2=1.6, \quad s_{2}=\sigma_{2}$ $(\mathbf{a b})+\sigma_{2}(\mathbf{b d})+\sigma_{2}(\mathbf{c d})+\sigma_{2}(\mathbf{d c})=0.3+0.3+0.1+0.2$ $=1.1 \quad$ and $\quad s_{3}=\tau(\mathbf{a b})+\tau(\mathbf{b d})+\tau(\mathbf{c d})+\tau(\mathbf{d c})=0.2+$ $0.3+0.1+0.2=0.8$. So, $S(\zeta)=(1.6,1.1,0.8)$.

Theorem 2 Let $\zeta=\left(V, E_{1}, E_{2}, \sigma_{1}, \sigma_{2}, \mu, \tau\right)$ be an IFMG such that there are one undirected edge and two opposite directed edges between every pair of vertices. If $|V|=n, n \in \mathbb{N}$, then

$v \leq \frac{o(\zeta)}{n} \leq 1$

$v \leq \frac{2 s_{1}}{n^{2}-n} \leq 1$

$v \leq \frac{s_{2}}{n^{2}-n} \leq 1$,

$0 \leq \frac{s_{3}}{n^{2}-n} \leq 1$

where $\quad v=\min \{\mu(a) ; a \in V\}, \quad o(\zeta)=$ order of $\zeta$, $\left(s_{1}, s_{2}, s_{3}\right)=S(\zeta)=$ size of $\zeta$.

Proof Since $v=\min \{\mu(a) ; a \in V\}, \quad$ so $\quad v \leq \mu(a) \leq 1$, $\forall a \in V$. 
Now, $|V|=n$, so $n v \leq \sum_{a \in V} \mu(a) \leq n \Rightarrow n v \leq \sum_{a \in V}$ $\mu(a) \leq n$

$\Rightarrow n v \leq o(\zeta) \leq n \Rightarrow v \leq \frac{o(\zeta)}{n} \leq 1$.

There is an undirected edge between every pair of vertices and $|V|=n$, so the total number of undirected edges in $\zeta$ is ${ }^{n} C_{2}$.

Now, $\quad \sigma_{1}(a b) \geq \mu(a) \wedge \mu(b) \geq \min \{\mu(a) ; a \in V\}=v$ and $\sigma_{1}(a b) \leq 1, \forall a b \in E_{1}$.

Then

$v \leq \sigma_{1}(a b) \leq 1, \forall a b \in E_{1} \Rightarrow v^{n} C_{2} \leq \sum_{a b \in E_{1}} \sigma_{1}(a b) \leq{ }^{n} C_{2}$

$\Rightarrow v \frac{n^{2}-n}{2} \leq s_{1} \leq \frac{n^{2}-n}{2} \Rightarrow v \leq \frac{2 s_{1}}{n^{2}-n} \leq 1$.

There are two opposite directed edges between every pair of vertices and $|V|=n$, so the total number of directed edges in $\zeta$ is $2^{n} C_{2}$.

Now, $\quad \sigma_{2}(\mathbf{a b}) \geq \mu(a) \wedge \mu(b) \geq \min \{\mu(a) ; a \in V\}=v$ and $\sigma_{2}(\mathbf{a b}) \leq 1, \forall \mathbf{a b} \in E_{2}$.

Then $\quad v \leq \sigma_{2}(\mathbf{a b}) \leq 1, \forall \mathbf{a b} \in E_{2} \Rightarrow 2 v^{n} C_{2} \leq \sum_{\mathbf{a b} \in E_{2}}$ $\sigma_{2}(\mathbf{a b}) \leq 2^{n} C_{2}$

$\Rightarrow v\left(n^{2}-n\right) \leq s_{2} \leq\left(n^{2}-n\right) \Rightarrow v \leq \frac{s_{2}}{n^{2}-n} \leq 1$.

In the Definition of IFMG we see that, $\tau(\mathbf{a b}) \geq \mid \mu(a)-$ $\mu(b) \mid \geq 0$ and

$\tau(\mathbf{a b}) \leq 1, \forall \mathbf{a b} \in E_{2}$.

So,

$0 \leq \tau(\mathbf{a b}) \leq 1, \forall \mathbf{a b} \in E_{2} \Rightarrow 0 \leq \sum_{\mathbf{a b} \in E_{2}} \tau(\mathbf{a b}) \leq 2^{n} C_{2}$

$\Rightarrow 0 \leq s_{3} \leq\left(n^{2}-n\right) \Rightarrow 0 \leq \frac{s_{3}}{n^{2}-n} \leq 1$.

Definition 10 The degree of a vertex ' $a$ ' of an IFMG $\zeta=$ $\left(V, E_{1}, E_{2}, \sigma_{1}, \sigma_{2}, \mu, \tau\right)$ is denoted by $d_{\text {ifmg }}(a)$ and is defined as

$$
\begin{gathered}
d_{\mathrm{ifmg}}(a)=\sum_{\substack{b \in V \\
a b \in E_{1}}} \sigma_{1}(a b)+\sum_{\substack{b \in V \\
\mathbf{b a} \in E_{2}}} \sigma_{2}(\mathbf{b a}) \\
-\sum_{\substack{b \in V \\
\mathbf{a b} \in E_{2}}} \sigma_{2}(\mathbf{a b}) .
\end{gathered}
$$

Example 5 Consider the IFMG $\zeta$ of Example 1. Here, $d_{\text {ifmg }}(d)=\left(\sigma_{1}(a d)+\sigma_{1}(b d)+\sigma_{1}(d c)\right)+\left(\sigma_{2}(\mathbf{b d})+\sigma_{2}\right.$ (cd) $) \quad-\left(\sigma_{2}(\mathbf{d c})\right)=(0.4+0.4+0.2)+(0.3+0.3)-$ $(0.2)=1.4$

Definition 11 Let $\zeta=\left(V, E_{1}, E_{2}, \sigma_{1}, \sigma_{2}, \mu, \tau\right)$ and $\zeta^{\prime}=$ $\left(V^{\prime}, E_{1}^{\prime}, E_{2}^{\prime}, \sigma_{1}^{\prime}, \sigma_{2}^{\prime}, \mu^{\prime}, \tau^{\prime}\right)$ be two IFMGs. Then $\zeta$ and $\zeta^{\prime}$ are isomorphic to each other if there exists a bijective mapping $f: \zeta \rightarrow \zeta^{\prime}$ such that $\mu(a)=\mu^{\prime}(f(a)), \forall a \in V, \sigma_{1}(a b)=$ $\sigma_{1}^{\prime}(f(a) f(b)), \sigma_{2}(\mathbf{a b})=\sigma_{2}^{\prime}(\mathbf{f}(\mathbf{a}) \mathbf{f}(\mathbf{b})), \tau(\mathbf{a b})=\tau^{\prime}(\mathbf{f}(\mathbf{a})$ $\mathbf{f}(\mathbf{b})), \forall a b \in E_{1}^{\prime}, \forall \mathbf{a b} \in E_{2}^{\prime}$.
Theorem 3 If two IFMGs $\zeta=\left(V, E_{1}, E_{2}, \sigma_{1}, \sigma_{2}, \mu, \tau\right)$ and $\zeta^{\prime}=\left(V^{\prime}, E_{1}^{\prime}, E_{2}^{\prime}, \sigma_{1}^{\prime}\right.$, $\left.\sigma_{2}^{\prime}, \mu^{\prime}, \tau^{\prime}\right)$ are isomorphic to each other, then

(i) $o(\zeta)=o\left(\zeta^{\prime}\right), S(\zeta)=S\left(\zeta^{\prime}\right)$

(ii) $\zeta^{c} \cong \zeta^{\prime c}$.

Proof Since $\zeta \cong \zeta^{\prime}$, so there is a bijective mapping $f$ from $\zeta$ to $\zeta^{\prime}$ such that $\mu(a)=\mu^{\prime}(f(a))=\mu^{\prime}\left(a^{\prime}\right), \forall a \in V$ and $\sigma_{1}(a b)=\sigma_{1}^{\prime}\left(a^{\prime} b^{\prime}\right), \forall a b \in E_{1}^{\prime}, \sigma_{2}(\mathbf{a b})=\sigma_{2}^{\prime}\left(\mathbf{a}^{\prime} \mathbf{b}^{\prime}\right), \tau(\mathbf{a b})=$ $\tau^{\prime}\left(\mathbf{a}^{\prime} \mathbf{b}^{\prime}\right), \forall \mathbf{a b} \in E_{2}^{\prime}$.

(i) $\sum_{a \in V} \mu(a)=\sum_{a^{\prime} \in V} \mu\left(a^{\prime}\right) \Rightarrow o(\zeta)=o\left(\zeta^{\prime}\right)$,

$\sum_{a b \in E_{1}} \sigma_{1}(a b)=\sum_{a^{\prime} b^{\prime} \in E_{2}} \sigma_{1}\left(a^{\prime} b^{\prime}\right), \quad \sum_{\mathbf{a b} \in E_{2}} \sigma_{2}((\mathbf{a b})=$ $\sum_{\mathbf{a}^{\prime} \mathbf{b}^{\prime} \in E_{2}} \sigma_{2}\left({ }^{\prime} \mathbf{b}^{\prime}\right), \quad \sum_{\mathbf{a b} \in E_{2}} \tau(a b)=\sum_{\mathbf{a}^{\prime} \mathbf{b}^{\prime} \in E_{2}} \sigma_{2}\left({ }^{\prime} \mathbf{b}^{\prime}\right)$. Hence, $S(\zeta)=S\left(\zeta^{\prime}\right)$.

(ii) From Definitions 8 and 11, we have $\mu^{c}(a)=$ $\mu(a), \forall a \in V, \mu^{\prime c}\left(a^{\prime}\right)=\mu^{\prime}\left(a^{\prime}\right), \forall a^{\prime} \in V^{\prime}$.

Now, $\quad \sigma_{1}^{c}(a b)=1-\sigma_{1}(a b)+\mu(a) \wedge \mu(b)=1-\sigma_{1}^{\prime}$ $\left(a^{\prime} b^{\prime}\right)+\mu^{\prime}\left(a^{\prime}\right) \wedge \mu^{\prime}\left(b^{\prime}\right)={\sigma^{\prime}}_{1}^{c}(a b), \sigma_{2}^{c}(\mathbf{a b})=1-\sigma_{2}(\mathbf{a b})+$ $\mu(a) \wedge \mu(b)=1-\sigma^{\prime}{ }_{2}\left(\mathbf{a}^{\prime} \mathbf{b}^{\prime}\right)+\mu^{\prime}\left(a^{\prime}\right) \wedge \mu^{\prime}\left(b^{\prime}\right), \tau^{c}(\mathbf{a b})=$ $1-\tau(\mathbf{a b})+|\mu(a)-\mu(b)|=1-\tau^{\prime}\left(\mathbf{a}^{\prime} \mathbf{b}^{\prime}\right)+\left|\mu^{\prime}\left(a^{\prime}\right)-\mu^{\prime}\left(b^{\prime}\right)\right|$.

All of these true $\forall a b \in E_{1}^{c}, \forall a^{\prime} b^{\prime} \in E_{1}^{\prime c}, \forall \mathbf{a b}$ $\in E_{2}^{c}, \forall \mathbf{a}^{\prime} \mathbf{b}^{\prime} \in E_{2}^{\prime c}$. Then, $\sigma_{1}(a b)=\sigma_{1}^{\prime}(f(a b))=\sigma_{1}^{\prime}\left(a^{\prime} b^{\prime}\right)$, $\sigma_{2}\left((\mathbf{a b})=\sigma_{2}^{\prime}(f(\mathbf{a b}))=\sigma_{1}^{\prime}\left(\left(\mathbf{a}^{\prime} \mathbf{b}^{\prime}\right)\right.\right.$,

$\tau\left((\mathbf{a b})=\tau^{\prime}(f(\mathbf{a b}))=\tau^{\prime}\left(\left(\mathbf{a}^{\prime} \mathbf{b}^{\prime}\right)\right.\right.$.

Hence, $\zeta^{c} \cong \zeta^{\prime c}$.

\section{Operations on inverse fuzzy mixed graphs}

In this section, we have defined union, intersection and join of two IFMGs. Then it is shown that the union, intersection and join of two IFMGs are again an IFMG. Cartesian product of two IFMGs is also defined here.

Definition 12 Let $\zeta=\left(V, E_{1}, E_{2}, \sigma_{1}, \sigma_{2}, \mu, \tau\right)$ and $\zeta^{\prime}=$ $\left(V^{\prime}, E_{1}^{\prime}, E_{2}^{\prime}, \sigma_{1}^{\prime}, \sigma_{2}^{\prime}, \mu^{\prime}, \tau^{\prime}\right)$ be two IFMGs. Then the Cartesian product of $\zeta$ and $\zeta^{\prime}$ is denoted by $\zeta \times \zeta^{\prime}$ and is defined as $\zeta \times \zeta^{\prime}=\left(A, B_{1}, B_{2}, \sigma_{1} \times \sigma_{1}^{\prime}, \sigma_{2} \times \sigma_{2}^{\prime}, \mu \times \mu^{\prime}, \tau \times \tau^{\prime}\right)$, where $A=V \times V^{\prime}=\left\{\left(a, a^{\prime}\right) ; a \in V, a^{\prime} \in V^{\prime}\right\}$,

$B_{1}=\left\{\left(a, b^{\prime}\right)\left(a, c^{\prime}\right) ; a \in V, b^{\prime} c^{\prime} \in E_{1}^{\prime}\right\} \quad \cup\left\{\left(b, a^{\prime}\right)\left(c, a^{\prime}\right) ;\right.$ $\left.b c \in E_{1}, a^{\prime} \in V^{\prime}\right\}$,

$B_{2}=\left\{\left(\mathbf{a}, \mathbf{b}^{\prime}\right)\left(\mathbf{a}, \mathbf{c}^{\prime}\right) ; a \in V, \mathbf{b}^{\prime} \mathbf{c}^{\prime} \in E_{2}^{\prime}\right\} \cup\left\{\left(\mathbf{b}, \mathbf{a}^{\prime}\right)\left(\mathbf{c}, \mathbf{a}^{\prime}\right) ;\right.$ bc $\left.\in E_{2}, a^{\prime} \in V^{\prime}\right\}$,

$\left(\mu \times \mu^{\prime}\right)\left(a, a^{\prime}\right)=\min \left\{\mu(a), \mu^{\prime}\left(a^{\prime}\right)\right\}, \forall\left(a, a^{\prime}\right) \in A$,

$\left(\sigma_{1} \times \sigma_{1}^{\prime}\right)\left(a, b^{\prime}\right)\left(a, c^{\prime}\right)=\min \left\{\mu(a), \sigma_{1}^{\prime}\left(b^{\prime} c^{\prime}\right)\right\}$,

$\forall a \in V, b^{\prime} c^{\prime} \in E_{1}^{\prime}$,

$\left(\sigma_{1} \times \sigma_{1}^{\prime}\right)\left(b, a^{\prime}\right)\left(c, a^{\prime}\right)=\min \left\{\sigma_{1}(b c), \mu^{\prime}\left(a^{\prime}\right)\right\}$,

$\forall b c \in E_{1}, a^{\prime} \in V^{\prime}$,

$\left(\sigma_{2} \times \sigma_{2}^{\prime}\right)\left(\mathbf{a}, \mathbf{b}^{\prime}\right)\left(\mathbf{a}, \mathbf{c}^{\prime}\right)=\min \left\{\mu(a), \sigma_{2}^{\prime}\left(\mathbf{b}^{\prime} \mathbf{c}^{\prime}\right)\right\}$,

$\forall a \in V, \mathbf{b}^{\prime} \mathbf{c}^{\prime} \in E_{2}^{\prime}$, 
$\left(\sigma_{2} \times \sigma_{2}^{\prime}\right)\left(\mathbf{b}, \mathbf{a}^{\prime}\right)\left(\mathbf{c}, \mathbf{a}^{\prime}\right)=\min \left\{\sigma_{2}(\mathbf{b c}), \mu^{\prime}\left(a^{\prime}\right)\right\}$,

$\forall \mathbf{b c} \in E_{2}, a^{\prime} \in V^{\prime}$,

$\left(\tau \times \tau^{\prime}\right)\left(\mathbf{a}, \mathbf{b}^{\prime}\right)\left(\mathbf{a}, \mathbf{c}^{\prime}\right)=\left|\mu(a)-\sigma_{2}^{\prime}\left(\mathbf{b}^{\prime} \mathbf{c}^{\prime}\right)\right|$,

$\forall a \in V, \mathbf{b}^{\prime} \mathbf{c}^{\prime} \in E_{2}^{\prime}$,

$\left(\tau \times \tau^{\prime}\right)\left(\mathbf{b}, \mathbf{a}^{\prime}\right)\left(\mathbf{c}, \mathbf{a}^{\prime}\right)=\left|\mu^{\prime}(a)-\sigma_{2}(\mathbf{b c})\right|$,

$\forall \mathbf{b c} \in E_{2}, a^{\prime} \in V^{\prime}$.

Theorem 4 Let $\zeta=\left(V, E_{1}, E_{2}, \sigma_{1}, \sigma_{2}, \mu, \tau\right)$ and $\zeta^{\prime}=$ $\left(V^{\prime}, E_{1}^{\prime}, E_{2}^{\prime}, \sigma_{1}^{\prime}, \sigma_{2}^{\prime}, \mu^{\prime}, \tau^{\prime}\right)$ be two IFMGs. Then $\zeta \times \zeta^{\prime}$ is also an IFMG.

Proof Let $a \in V, b^{\prime} c^{\prime} \in E_{1}^{\prime}$. Then

$\left(\sigma_{1} \times \sigma_{1}^{\prime}\right)\left(a, b^{\prime}\right)\left(a, c^{\prime}\right)=\min \left\{\mu(a), \sigma_{1}^{\prime}\left(b^{\prime} c^{\prime}\right)\right\} \geq \min \{\mu(a)$, $\left.\min \left\{\mu^{\prime}\left(b^{\prime}\right), \mu^{\prime}\left(c^{\prime}\right)\right\}\right\}$

$=\min \left\{\min \left\{\mu(a), \mu^{\prime}\left(b^{\prime}\right)\right\}, \min \left\{\mu(a), \mu^{\prime}\left(c^{\prime}\right)\right\}\right\}$

$=\min \left\{\left(\mu \times \mu^{\prime}\right)\left(a, b^{\prime}\right),\left(\mu \times \mu^{\prime}\right)\left(a, c^{\prime}\right)\right\}$.

Therefore, $\quad\left(\sigma_{1} \times \sigma_{1}^{\prime}\right)\left(a, b^{\prime}\right)\left(a, c^{\prime}\right) \geq \min \left\{\left(\mu \times \mu^{\prime}\right)\left(a, b^{\prime}\right)\right.$, $\left.\left(\mu \times \mu^{\prime}\right)\left(a, c^{\prime}\right)\right\}, \forall a \in V, b^{\prime} c^{\prime} \in E_{1}^{\prime}$.

Similarly, $\quad\left(\sigma_{1} \times \sigma_{1}^{\prime}\right)\left(b, a^{\prime}\right)\left(c, a^{\prime}\right) \geq \min \left\{\left(\mu \times \mu^{\prime}\right)\right.$ $\left.\left(b, a^{\prime}\right),\left(\mu \times \mu^{\prime}\right)\left(c, a^{\prime}\right)\right\}, \forall b c \in E_{1}, a^{\prime} \in V^{\prime}$.

$\left(\sigma_{2} \times \sigma_{2}^{\prime}\right)\left(\mathbf{a}, \mathbf{b}^{\prime}\right)\left(\mathbf{a}, \mathbf{c}^{\prime}\right)=\min \left\{\mu(a), \sigma_{2}^{\prime}\left(\mathbf{b}^{\prime} \mathbf{c}^{\prime}\right)\right\} \geq \min \{\mu(a)$ , $\left.\min \left\{\mu^{\prime}\left(b^{\prime}\right), \mu^{\prime}\left(c^{\prime}\right)\right\}\right\}$

$=\min \left\{\min \left\{\mu(a), \mu^{\prime}\left(b^{\prime}\right)\right\}, \min \left\{\mu(a), \mu^{\prime}\left(c^{\prime}\right)\right\}\right\}$

$=\min \left\{\left(\mu \times \mu^{\prime}\right)\left(a, b^{\prime}\right),\left(\mu \times \mu^{\prime}\right)\left(a, c^{\prime}\right)\right\}$.

Therefore, $\quad\left(\sigma_{2} \times \sigma_{2}^{\prime}\right)\left(\mathbf{a}, \mathbf{b}^{\prime}\right)\left(\mathbf{a}, \mathbf{c}^{\prime}\right) \geq \min \left\{\left(\mu \times \mu^{\prime}\right)\left(a, b^{\prime}\right)\right.$, $\left.\left(\mu \times \mu^{\prime}\right)\left(a, c^{\prime}\right)\right\}, \forall a \in V, \mathbf{b}^{\prime} \mathbf{c}^{\prime} \in E_{2}^{\prime}$.

Similarly, $\left(\sigma_{2} \times \sigma_{2}^{\prime}\right)\left(\mathbf{b}, \mathbf{a}^{\prime}\right)\left(\mathbf{c}, \mathbf{a}^{\prime}\right) \geq \min \left\{\left(\mu \times \mu^{\prime}\right)\left(b, a^{\prime}\right)\right.$, $\left.\left(\mu \times \mu^{\prime}\right)\left(c, a^{\prime}\right)\right\}, \forall \mathbf{b c} \in E_{2}, a^{\prime} \in V^{\prime}$.

$\left(\tau \times \tau^{\prime}\right)\left(\mathbf{a}, \mathbf{b}^{\prime}\right)\left(\mathbf{a}, \mathbf{c}^{\prime}\right)=\left|\mu(a)-\sigma_{2}^{\prime}\left(\mathbf{b}^{\prime} \mathbf{c}^{\prime}\right)\right|$

$\geq\left|\left(\mu \times \mu^{\prime}\right)\left(a, b^{\prime}\right)-\left(\mu \times \mu^{\prime}\right)\left(a, c^{\prime}\right)\right|, \forall a \in V, \mathbf{b}^{\prime} \mathbf{c}^{\prime} \in E_{2}^{\prime}$.

Similarly, $\left(\tau \times \tau^{\prime}\right)\left(\mathbf{b}, \mathbf{a}^{\prime}\right)\left(\mathbf{c}, \mathbf{a}^{\prime}\right) \geq \mid\left(\mu \times \mu^{\prime}\right)\left(b, a^{\prime}\right)-(\mu \times$ $\left.\mu^{\prime}\right)\left(c, a^{\prime}\right) \mid, \forall \mathbf{b c} \in E_{2}, a^{\prime} \in V^{\prime}$.

Now, $\zeta \times \zeta^{\prime}$ satisfies all the conditions of an IFMG. Therefore, $\zeta \times \zeta^{\prime}$ is an IFMG.

Example 6 Consider the IFMGs $\zeta$ and $\zeta^{\prime}$ of Fig. 3. Here, $\left(\mu \times \mu^{\prime}\right)\left(a, b^{\prime}\right)=\min \left\{\mu(a), \mu^{\prime}\left(b^{\prime}\right)\right\}=0.3$,

$\left(\mu \times \mu^{\prime}\right)\left(b, b^{\prime}\right)=\min \left\{\mu(b), \mu^{\prime}\left(b^{\prime}\right)\right\}=0.3$.

$\left(\sigma_{1} \times \sigma_{1}^{\prime}\right)\left(a, b^{\prime}\right)\left(b, b^{\prime}\right)=\min \left\{\mu\left(b^{\prime}\right), \sigma_{1}(a b)\right\}=0.3$,

$\min \left\{\left(\mu \times \mu^{\prime}\right)\left(a, b^{\prime}\right),\left(\mu \times \mu^{\prime}\right)\left(b, c^{\prime}\right)\right\}=0.3$,

$\left(\sigma_{2} \times \sigma_{2}^{\prime}\right)\left(\mathbf{a}, \mathbf{b}^{\prime}\right)\left(\mathbf{b}, \mathbf{b}^{\prime}\right)=\min \left\{\mu\left(b^{\prime}\right), \sigma_{2}(\mathbf{a b})\right\}=0.3$,

$\left(\tau \times \tau^{\prime}\right)\left(\mathbf{a}, \mathbf{b}^{\prime}\right)\left(\mathbf{b}, \mathbf{b}^{\prime}\right)=\left|\mu\left(b^{\prime}\right)-\sigma_{2}^{\prime}(\mathbf{a b})\right|=0.5$,

$\left|\left(\mu \times \mu^{\prime}\right)\left(a, b^{\prime}\right)-\left(\mu \times \mu^{\prime}\right)\left(b, b^{\prime}\right)\right|=0$.

So, $\left(\sigma_{1} \times \sigma_{1}^{\prime}\right)\left(a, b^{\prime}\right)\left(b, b^{\prime}\right) \geq \min \left\{\left(\mu \times \mu^{\prime}\right)\left(a, b^{\prime}\right),(\mu \times\right.$ $\left.\left.\mu^{\prime}\right)\left(b, c^{\prime}\right)\right\}$, $\left(\sigma_{2} \times \sigma_{2}^{\prime}\right)\left(\mathbf{a}, \mathbf{b}^{\prime}\right)\left(\mathbf{b}, \mathbf{b}^{\prime}\right) \geq \min \left\{\left(\mu \times \mu^{\prime}\right)\left(a, b^{\prime}\right),\left(\mu \times \mu^{\prime}\right)\left(b, c^{\prime}\right)\right\}$

and

$\left(\tau \times \tau^{\prime}\right)\left(\mathbf{a}, \mathbf{b}^{\prime}\right)\left(\mathbf{b}, \mathbf{b}^{\prime}\right) \geq\left|\left(\mu \times \mu^{\prime}\right)\left(a, b^{\prime}\right)-\left(\mu \times \mu^{\prime}\right)\left(b, b^{\prime}\right)\right|$.

Thus, the edge $\left(a, b^{\prime}\right)\left(b, b^{\prime}\right)$ and $\left(\mathbf{a}, \mathbf{b}^{\prime}\right)\left(\mathbf{b}, \mathbf{b}^{\prime}\right)$ of $\zeta \times \zeta^{\prime}$ satisfies all the relations of an IFMG. Similarly, all the others edges of $\zeta \times \zeta^{\prime}$ satisfies all the relations of an IFMG. Hence, $\zeta \times \zeta^{\prime}$ is an IFMG.

Definition 13 Let $\zeta=\left(V, E_{1}, E_{2}, \sigma_{1}, \sigma_{2}, \mu, \tau\right)$ and $\zeta^{\prime}=$ $\left(V^{\prime}, E_{1}^{\prime}, E_{2}^{\prime}, \sigma_{1}^{\prime}, \sigma_{2}^{\prime}, \mu^{\prime}, \tau^{\prime}\right)$ be two IFMGs. Then the union of $\zeta$ and $\zeta^{\prime}$ is denoted by $\zeta \cup \zeta^{\prime}$ and is defined as

$\zeta \cup \zeta^{\prime}=\left(V \cup V^{\prime}, E_{1} \cup E_{1}^{\prime}, E_{2} \cup E_{2}^{\prime}, \sigma_{1} \cup \sigma_{1}^{\prime}, \sigma_{2} \cup \sigma_{2}^{\prime}, \mu \cup\right.$ $\left.\mu^{\prime}, \tau \cup \tau^{\prime}\right)$, where

$$
\begin{aligned}
& \left(\mu \cup \mu^{\prime}\right)(a)=\mu(a) \text { if } a \in V-V^{\prime} \\
& \left(\mu \cup \mu^{\prime}\right)(a)=\mu^{\prime}(a) \text { if } a \in V^{\prime}-V \\
& \left(\mu \cup \mu^{\prime}\right)(a)=\min \left\{\mu(a), \mu^{\prime}(a)\right\} \text { if } a \in V \cap V^{\prime} \\
& \left(\sigma_{1} \cup \sigma_{1}^{\prime}\right)(a b)=\sigma_{1}(a b) \text { if } a b \in E_{1}-E_{1}^{\prime} \\
& \left(\sigma_{1} \cup \sigma_{1}^{\prime}\right)(a b)=\sigma_{1}^{\prime}(a b) \text { if } a b \in E_{1}^{\prime}-E_{1} \\
& \left(\sigma_{1} \cup \sigma_{1}^{\prime}\right)(a b)=\max \left\{\sigma_{1}(a b), \sigma_{1}^{\prime}(a b)\right\} \text { if } a b \in E_{1} \cap E_{1}^{\prime} \\
& \left(\sigma_{2} \cup \sigma_{2}^{\prime}\right)(\mathbf{a b})=\sigma_{2}(\mathbf{a b}) \text { if } \mathbf{a b} \in E_{2}-E_{2}^{\prime} \\
& \left(\sigma_{2} \cup \sigma_{2}^{\prime}\right)(\mathbf{a b})=\sigma_{2}^{\prime}(\mathbf{a b}) \text { if } \mathbf{a b} \in E_{2}^{\prime}-E_{2} \\
& \left(\sigma_{2} \cup \sigma_{2}^{\prime}\right)(\mathbf{a b})=\max \left\{\sigma_{2}(\mathbf{a b}), \sigma_{2}^{\prime}(\mathbf{a b})\right\} \text { if } \mathbf{a b} \in E_{2} \cap E_{2}^{\prime} \\
& \left(\tau \cup \tau^{\prime}\right)(\mathbf{a b})=\tau(\mathbf{a b}) \text { if } \mathbf{a b} \in E_{2}-E_{2}^{\prime} \\
& \left(\tau \cup \tau^{\prime}\right)(\mathbf{a b})=\tau^{\prime}(\mathbf{a b}) \text { if } \mathbf{a b} \in E_{2}^{\prime}-E_{2} \\
& \left(\tau \cup \tau^{\prime}\right)(\mathbf{a b})=\max \left\{\tau(\mathbf{a b}), \tau^{\prime}(\mathbf{a b})\right\} \text { if } \mathbf{a b} \in E_{2} \cap E_{2}^{\prime} .
\end{aligned}
$$

Theorem 5 Let $\zeta=\left(V, E_{1}, E_{2}, \sigma_{1}, \sigma_{2}, \mu, \tau\right)$ and $\zeta^{\prime}=$ $\left(V^{\prime}, E_{1}^{\prime}, E_{2}^{\prime}, \sigma_{1}^{\prime}, \sigma_{2}^{\prime}, \mu^{\prime}, \tau^{\prime}\right)$ be two IFMGs. Then $\zeta \cup \zeta^{\prime}$ is also an IFMG.

Proof Case-I: Let $a b \in E_{1}$ and $\mathbf{a b} \in E_{2}$. Then

$$
\begin{aligned}
\left(\sigma_{1} \cup \sigma_{1}^{\prime}\right)(a b) & =\sigma_{1}(a b) \geq \min \{\mu(a), \mu(b)\} \\
& \geq \min \left\{\min \left\{\mu(a), \mu^{\prime}(a)\right\}, \min \left\{\mu(b), \mu^{\prime}(b)\right\}\right\} \\
& =\min \left\{\left(\mu \cup \mu^{\prime}\right)(a),\left(\mu \cup \mu^{\prime}\right)(b)\right\} . \\
\left(\sigma_{2} \cup \sigma_{2}^{\prime}\right)(\mathbf{a b}) & =\sigma_{2}(\mathbf{a b}) \geq \min \{\mu(a), \mu(b)\} \\
& \geq \min \left\{\min \left\{\mu(a), \mu^{\prime}(a)\right\}, \min \left\{\mu(b), \mu^{\prime}(b)\right\}\right\} \\
& =\min \left\{\left(\mu \cup \mu^{\prime}\right)(a),\left(\mu \cup \mu^{\prime}\right)(b)\right\} . \\
\left(\tau \cup \tau^{\prime}\right)(\mathbf{a b}) & =\tau(\mathbf{a b}) \geq|\mu(a)-\mu(b)| \\
& \geq\left|\left(\mu \cup \mu^{\prime}\right)(a)-\left(\mu \cup \mu^{\prime}\right)(b)\right| .
\end{aligned}
$$

Case-II: Let $a b \in E_{1}^{\prime}$ and $\mathbf{a b} \in E_{2}^{\prime}$. Then similarly as in Case-I, we have 


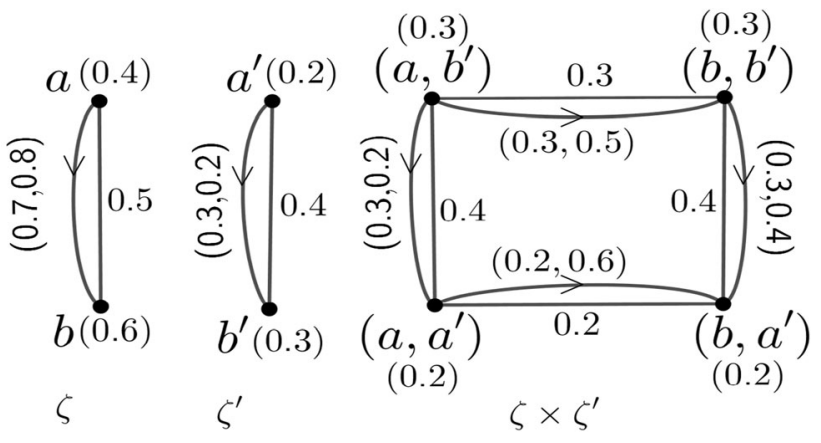

Fig. $3 \zeta \times \zeta^{\prime}$, the Cartesian product of two IFMGs $\zeta$ and $\zeta^{\prime}$

$$
\begin{aligned}
& \left(\sigma_{1} \cup \sigma_{1}^{\prime}\right)(a b) \geq \min \left\{\left(\mu \cup \mu^{\prime}\right)(a),\left(\mu \cup \mu^{\prime}\right)(b)\right\} . \\
& \left(\sigma_{2} \cup \sigma_{2}^{\prime}\right)(\mathbf{a b}) \geq \min \left\{\left(\mu \cup \mu^{\prime}\right)(a),\left(\mu \cup \mu^{\prime}\right)(b)\right\} . \\
& \text { and }\left(\tau \cup \tau^{\prime}\right)(\mathbf{a b}) \geq\left|\left(\mu \cup \mu^{\prime}\right)(a)-\left(\mu \cup \mu^{\prime}\right)(b)\right| .
\end{aligned}
$$

Case-III: Let $a b \in E_{1} \cap E_{1}^{\prime}$ and $\mathbf{a b} \in E_{2} \cap E_{2}^{\prime}$. Then

$$
\begin{aligned}
& \left(\sigma_{1} \cup \sigma_{1}^{\prime}\right)(a b)=\max \left\{\sigma_{1}(a b), \sigma_{1}^{\prime}(a b)\right\} \\
& \quad \geq \max \left\{\min \{\mu(a), \mu(b)\}, \min \left\{\mu^{\prime}(a), \mu^{\prime}(b)\right\}\right\} \\
& \quad \geq \min \left\{\min \left\{\mu(a), \mu^{\prime}(a)\right\}, \min \left\{\mu(b), \mu^{\prime}(b)\right\}\right\} \\
& \quad=\min \left\{\left(\mu \cup \mu^{\prime}\right)(a),\left(\mu \cup \mu^{\prime}\right)(b)\right\} . \\
& \left(\sigma_{2} \cup \sigma_{2}^{\prime}\right)(\mathbf{a b})=\max \left\{\sigma_{2}(\mathbf{a b}), \sigma_{2}^{\prime}(\mathbf{a b})\right\} \\
& \quad \geq \max \left\{\min \{\mu(a), \mu(b)\}, \min \left\{\mu^{\prime}(a), \mu^{\prime}(b)\right\}\right\} \\
& \quad \geq \min \left\{\min \left\{\mu(a), \mu^{\prime}(a)\right\}, \min \left\{\mu(b), \mu^{\prime}(b)\right\}\right\} \\
& \quad=\min \left\{\left(\mu \cup \mu^{\prime}\right)(a),\left(\mu \cup \mu^{\prime}\right)(b)\right\} . \\
& \left(\tau \cup \tau^{\prime}\right)(\mathbf{a b})=\max \left\{\tau(\mathbf{a b}), \tau^{\prime}(\mathbf{a b})\right\} \\
& \quad \geq \max \left\{|\mu(a)-\mu(b)|,\left|\mu^{\prime}(a)-\mu^{\prime}(b)\right|\right\} \\
& \quad=\left|\max \left\{\mu(a), \mu^{\prime}(a)\right\}-\max \left\{\mu(b), \mu^{\prime}(b)\right\}\right| \\
& \quad=\left|\left(\mu \cup \mu^{\prime}\right)(a)-\left(\mu \cup \mu^{\prime}\right)(b)\right| .
\end{aligned}
$$

Now, $\zeta \cup \zeta^{\prime}$ satisfies all the conditions of an IFMG. Therefore, $\zeta \cup \zeta^{\prime}$ is an IFMG.

Example 7 Consider the IFMGs $\zeta$ and $\zeta^{\prime}$ of Fig. 4.

Here, $\left(\sigma_{1} \cup \sigma_{1}^{\prime}\right)(a b)=0.5,\left(\sigma_{2} \cup \sigma_{2}^{\prime}\right)(\mathbf{a b})=0.7, \quad(\tau \cup$ $\left.\tau^{\prime}\right)(\mathbf{a b})=0.8, \min \left\{\left(\mu \cup \mu^{\prime}\right)(a),\left(\mu \cup \mu^{\prime}\right)(b)\right\}=0.2, \mid(\mu \cup$ $\left.\mu^{\prime}\right)(a)-\left(\mu \cup \mu^{\prime}\right)(b) \mid=0.4$.

So, $\left(\sigma_{1} \cup \sigma_{1}^{\prime}\right)(a b) \geq \min \left\{\left(\mu \cup \mu^{\prime}\right)(a),\left(\mu \cup \mu^{\prime}\right)(b)\right\}$, $\left(\sigma_{2} \cup \sigma_{2}^{\prime}\right)(\mathbf{a b}) \geq \min \left\{\left(\mu \cup \mu^{\prime}\right)(a),\left(\mu \cup \mu^{\prime}\right)(b)\right\}$ and $\left(\tau \cup \tau^{\prime}\right)(\mathbf{a b}) \geq\left|\left(\mu \cup \mu^{\prime}\right)(a)-\left(\mu \cup \mu^{\prime}\right)(b)\right|$.

Thus, the edge $a b$ and ab of $\zeta \cup \zeta^{\prime}$ satisfies all the relations of an IFMG. Similarly, all the others edges of $\zeta \cup \zeta^{\prime}$ satisfies all the relations of an IFMG. Hence, $\zeta \cup \zeta^{\prime}$ is an IFMG.

Definition 14 Let $\zeta=\left(V, E_{1}, E_{2}, \sigma_{1}, \sigma_{2}, \mu, \tau\right)$ and $\zeta^{\prime}=$ $\left(V^{\prime}, E_{1}^{\prime}, E_{2}^{\prime}, \sigma_{1}^{\prime}, \sigma_{2}^{\prime}, \mu^{\prime}, \tau^{\prime}\right)$ be two IFMGs. Then the intersection of $\zeta$ and $\zeta^{\prime}$ is denoted by $\zeta \cap \zeta^{\prime}$ and is defined as

$\zeta \cap \zeta^{\prime}=\left(V \cap V^{\prime}, E_{1} \cap E_{1}^{\prime}, E_{2} \cap E_{2}^{\prime}, \sigma_{1} \cap \sigma_{1}^{\prime}, \sigma_{2} \cap \sigma_{2}^{\prime}, \mu\right.$ $\left.\cap \mu^{\prime}, \tau \cap \tau^{\prime}\right)$, where $\left(\mu \cap \mu^{\prime}\right)(a)=\min \left\{\mu(a), \mu^{\prime}(a)\right\}$ if $a \in V \cap V^{\prime}$

$\left(\sigma_{1} \cap \sigma_{1}^{\prime}\right)(a b)=\min \left\{\sigma_{1}(a b), \sigma_{1}^{\prime}(a b)\right\}$ if $a b \in E_{1} \cap E_{1}^{\prime}$

$\left(\sigma_{2} \cap \sigma_{2}^{\prime}\right)(\mathbf{a b})=\min \left\{\sigma_{2}(\mathbf{a b}), \sigma_{2}^{\prime}(\mathbf{a b})\right\}$ if $\mathbf{a b} \in E_{2} \cap E_{2}^{\prime}$

$\left(\tau \cap \tau^{\prime}\right)(\mathbf{a b})=\min \left\{\tau(\mathbf{a b}), \tau^{\prime}(\mathbf{a b})\right\}$ if $\mathbf{a b} \in E_{2} \cap E_{2}^{\prime}$.

Theorem 6 Let $\zeta=\left(V, E_{1}, E_{2}, \sigma_{1}, \sigma_{2}, \mu, \tau\right)$ and $\zeta^{\prime}=$ $\left(V^{\prime}, E_{1}^{\prime}, E_{2}^{\prime}, \sigma_{1}^{\prime}, \sigma_{2}^{\prime}, \mu^{\prime}, \tau^{\prime}\right)$ be two IFMGs. Then $\zeta \cap \zeta^{\prime}$ is also an IFMG.

Proof Let $a b \in E_{1} \cap E_{1}^{\prime}$ and $\mathbf{a b} \in E_{2} \cap E_{2}^{\prime}$. Then

$$
\begin{aligned}
& \left(\sigma_{1} \cap \sigma_{1}^{\prime}\right)(a b)=\min \left\{\sigma_{1}(a b), \sigma_{1}^{\prime}(a b)\right\} \\
& \geq \min \left\{\min \{\mu(a), \mu(b)\}, \min \left\{\mu^{\prime}(a), \mu^{\prime}(b)\right\}\right\} \\
& \geq \min \left\{\min \left\{\mu(a), \mu^{\prime}(a)\right\}, \min \left\{\mu(b), \mu^{\prime}(b)\right\}\right\} \\
& =\min \left\{\left(\mu \cap \mu^{\prime}\right)(a),\left(\mu \cap \mu^{\prime}\right)(b)\right\} . \\
& \left(\sigma_{2} \cap \sigma_{2}^{\prime}\right)(\mathbf{a b})=\min \left\{\sigma_{2}(\mathbf{a b}), \sigma_{2}^{\prime}(\mathbf{a b})\right\} \\
& \geq \min \left\{\min \{\mu(a), \mu(b)\}, \min \left\{\mu^{\prime}(a), \mu^{\prime}(b)\right\}\right\} \\
& \geq \min \left\{\min \left\{\mu(a), \mu^{\prime}(a)\right\}, \min \left\{\mu(b), \mu^{\prime}(b)\right\}\right\} \\
& =\min \left\{\left(\mu \cap \mu^{\prime}\right)(a),\left(\mu \cap \mu^{\prime}\right)(b)\right\} . \\
& \left(\tau \cap \tau^{\prime}\right)(\mathbf{a b})=\min \left\{\tau(\mathbf{a b}), \tau^{\prime}(\mathbf{a b})\right\} \\
& \geq \min \left\{|\mu(a)-\mu(b)|,\left|\mu^{\prime}(a)-\mu^{\prime}(b)\right|\right\} \\
& =\left|\left(\mu \cap \mu^{\prime}\right)(a)-\left(\mu \cap \mu^{\prime}\right)(b)\right| .
\end{aligned}
$$

Now, $\zeta \cap \zeta^{\prime}$ satisfies all the conditions of an IFMG. Therefore, $\zeta \cup \zeta^{\prime}$ is an IFMG.

Example 8 Consider the IFMGs $\zeta$ and $\zeta^{\prime}$ of Fig. 5.

Here, the vertices $a, b^{\prime}$ and the only undirected edge $a b^{\prime}$ belongs to $\zeta \cap \zeta^{\prime} . \quad\left(\sigma_{1} \cap \sigma_{1}^{\prime}\right)(a b)=\min \left\{\sigma_{1}\left(a b^{\prime}\right)\right.$, $\left.\sigma_{1}^{\prime}\left(a b^{\prime}\right)\right\}=0.3, \quad \min \left\{\left(\mu \cap \mu^{\prime}\right)(a),\left(\mu \cap \mu^{\prime}\right)(b)\right\}=0.2$. So, $\left(\sigma_{1} \cap \sigma_{1}^{\prime}\right)(a b) \geq \min \left\{\left(\mu \cap \mu^{\prime}\right)(a),\left(\mu \cap \mu^{\prime}\right)(b)\right\}$. Hence, $\zeta \cup$ $\zeta^{\prime}$ is an IFMG.

Definition 15 Let $\zeta=\left(V, E_{1}, E_{2}, \sigma_{1}, \sigma_{2}, \mu, \tau\right)$ and $\zeta^{\prime}=$ $\left(V^{\prime}, E_{1}^{\prime}, E_{2}^{\prime}, \sigma_{1}^{\prime}, \sigma_{2}^{\prime}, \mu^{\prime}, \tau^{\prime}\right)$ be two IFMGs. Then the join of $\zeta$ and $\zeta^{\prime}$ is denoted by $\zeta+\zeta^{\prime}$ and is defined as
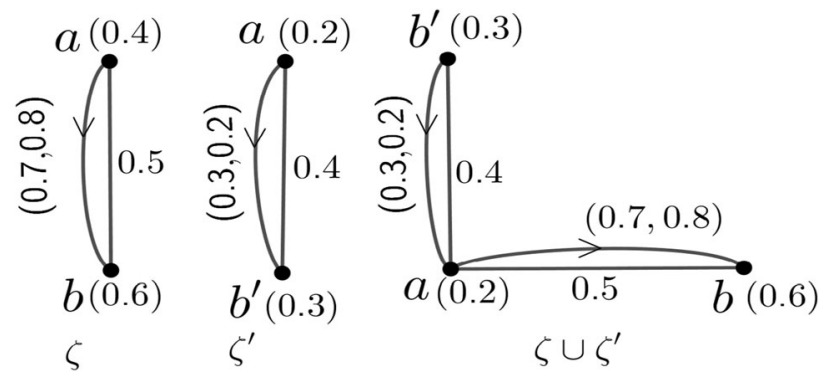

Fig. $4 \zeta \cup \zeta^{\prime}$, the union of two IFMGs $\zeta$ and $\zeta^{\prime}$ 
$\zeta \cup \zeta^{\prime}=\left(V \cup V^{\prime}, E_{1} \cup E_{1}^{\prime} \cup E_{1}^{*}, E_{2} \cup E_{2}^{\prime} \cup E_{2}^{*}, \sigma_{1}+\right.$ $\left.\sigma_{1}^{\prime}, \sigma_{2}+\sigma_{2}^{\prime}, \mu+\mu^{\prime}, \tau+\tau^{\prime}\right)$, where

$\left(\mu+\mu^{\prime}\right)(a)=\left(\mu \cup \mu^{\prime}\right)(a), \forall a \in V \cup V^{\prime}$

$\left(\sigma_{1}+\sigma_{1}^{\prime}\right)(a b)=\left(\sigma_{1} \cup \sigma_{1}^{\prime}\right)(a b)$ if $a b \in E_{1} \cup E_{1}^{\prime}$

$\left(\sigma_{1}+\sigma_{1}^{\prime}\right)(a b)=\max \left\{\mu(a), \mu^{\prime}(b)\right\}$ if $a b \in E_{1}^{*}$

$\left(\sigma_{2}+\sigma_{2}^{\prime}\right)(\mathbf{a b})=\left(\sigma_{2} \cup \sigma_{1}^{\prime}\right)(\mathbf{a b})$ if $\mathbf{a b} \in E_{2} \cup E_{2}^{\prime}$

$\left(\sigma_{2}+\sigma_{2}^{\prime}\right)(\mathbf{a b})=\max \left\{\mu(a), \mu^{\prime}(b)\right\}$ if $\mathbf{a b} \in E_{2}^{*}$

$\left(\tau+\tau^{\prime}\right)(\mathbf{a b})=\left(\tau \cup \tau^{\prime}\right)(\mathbf{a b})$ if $\mathbf{a b} \in E_{2} \cup E_{2}^{\prime}$

$\left(\tau+\tau^{\prime}\right)(\mathbf{a b})=\max \left\{\mu(a), \mu^{\prime}(b)\right\}$ if $\mathbf{a b} \in E_{2}^{*}$,

where $E_{1}^{*}$ and $E_{2}^{*}$ are respectively set of all undirected and directed edges joining all the distinct vertices of $V$ and $V^{\prime}$.

Theorem 7 Let $\zeta=\left(V, E_{1}, E_{2}, \sigma_{1}, \sigma_{2}, \mu, \tau\right)$ and $\zeta^{\prime}=$ $\left(V^{\prime}, E_{1}^{\prime}, E_{2}^{\prime}, \sigma_{1}^{\prime}, \sigma_{2}^{\prime}, \mu^{\prime}, \tau^{\prime}\right)$ be two IFMGs. Then $\zeta+\zeta^{\prime}$ is also an IFMG.

Proof Case-I: Let $a b \in E_{1} \cup E_{1}^{\prime}$ and $\mathbf{a b} \in E_{2} \cup E_{2}^{\prime}$. Then by the Theorem 5 , we have

$$
\begin{aligned}
& \left(\sigma_{1}+\sigma_{1}^{\prime}\right)(a b)=\left(\sigma_{1} \cup \sigma_{1}^{\prime}\right)(a b) \\
& \geq \min \left\{\left(\mu \cup \mu^{\prime}\right)(a),\left(\mu \cup \mu^{\prime}\right)(b)\right\} \\
& =\min \left\{\left(\mu+\mu^{\prime}\right)(a),\left(\mu+\mu^{\prime}\right)(b)\right\} \\
& \left(\sigma_{2}+\sigma_{2}^{\prime}\right)(\mathbf{a b})=\left(\sigma_{2} \cup \sigma_{2}^{\prime}\right)(\mathbf{a b}) \\
& \geq \min \left\{\left(\mu \cup \mu^{\prime}\right)(a),\left(\mu \cup \mu^{\prime}\right)(b)\right\} \\
& =\min \left\{\left(\mu+\mu^{\prime}\right)(a),\left(\mu+\mu^{\prime}\right)(b)\right\} \\
& \left(\tau+\tau^{\prime}\right)(\mathbf{a b})=\left(\tau \cup \tau^{\prime}\right)(\mathbf{a b}) \geq\left|\left(\mu \cup \mu^{\prime}\right)(a)-\left(\mu \cup \mu^{\prime}\right)(b)\right| \\
& =\left|\left(\mu+\mu^{\prime}\right)(a)-\left(\mu+\mu^{\prime}\right)(b)\right| .
\end{aligned}
$$

Case-II: Let $a b \in E_{1}^{*}$ and $\mathbf{a b} \in E_{2}^{*}$. Then

$$
\begin{aligned}
& \left(\sigma_{1}+\sigma_{1}^{\prime}\right)(a b)=\max \left\{\mu(a), \mu^{\prime}(b)\right\} \geq \min \left\{\left(\mu \cup \mu^{\prime}\right)(a),\right. \\
& \left.\left(\mu \cup \mu^{\prime}\right)(b)\right\}=\min \left\{\left(\mu+\mu^{\prime}\right)(a),\left(\mu+\mu^{\prime}\right)(b)\right\} \\
& \left(\sigma_{2}+\sigma_{2}^{\prime}\right)(\mathbf{a b})=\max \left\{\mu(a), \mu^{\prime}(b)\right\} \geq \min \left\{\left(\mu \cup \mu^{\prime}\right)(a),\right. \\
& \left.\left(\mu \cup \mu^{\prime}\right)(b)\right\}=\min \left\{\left(\mu+\mu^{\prime}\right)(a),\right. \\
& \left.\left(\mu+\mu^{\prime}\right)(b)\right\}\left(\tau+\tau^{\prime}\right)(\mathbf{a b})=\max \left\{\mu(a), \mu^{\prime}(b)\right\} \\
& \quad \geq\left|\left(\mu \cup \mu^{\prime}\right)(a)-\left(\mu \cup \mu^{\prime}\right)(b)\right| \\
& =\left|\left(\mu+\mu^{\prime}\right)(a)-\left(\mu+\mu^{\prime}\right)(b)\right| .
\end{aligned}
$$
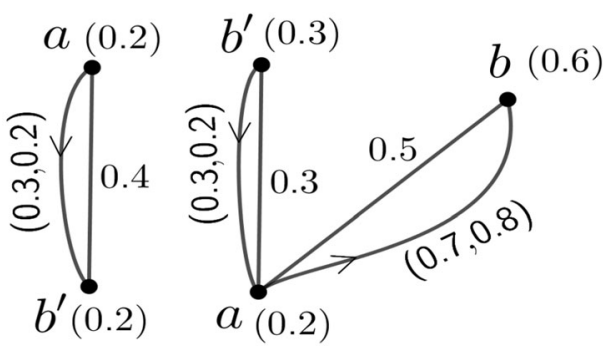

$\zeta$

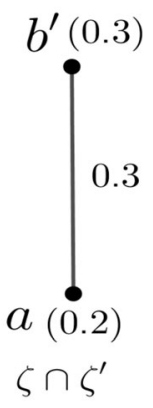

Fig. $5 \zeta \cap \zeta^{\prime}$, the intersection of two IFMGs $\zeta$ and $\zeta^{\prime}$
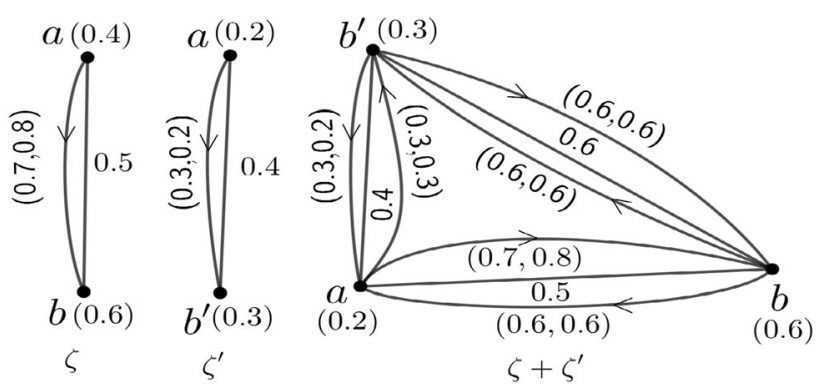

Fig. $6 \zeta+\zeta^{\prime}$, the join of two IFMGs $\zeta$ and $\zeta^{\prime}$ 


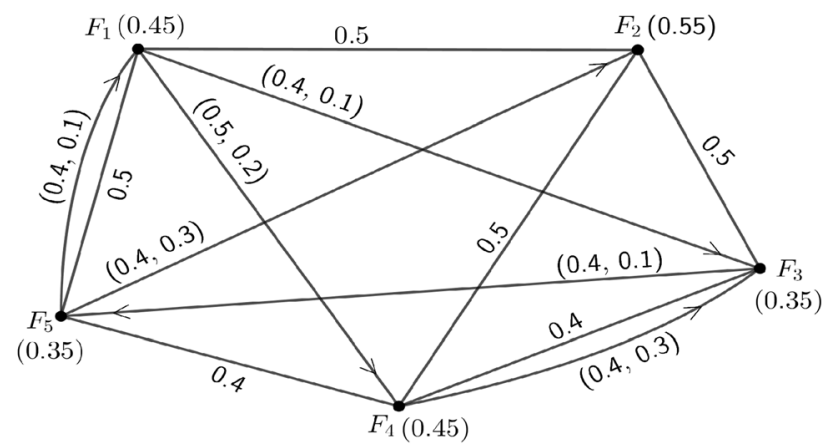

Fig. 7 An IMFG $\zeta$ corresponding to the communication gap of five friends

$$
\begin{aligned}
& \sum_{b \in V} \quad \sigma_{1}(a b)+\sum b \in V \quad \sigma_{2}(\mathbf{b a}) \\
& a b \in E_{1} \quad \text { ba } \in E_{2} \\
& -\sum b \in V \quad \sigma_{2}(\mathbf{a b}) \text {. } \\
& \mathbf{a b} \in E_{2}
\end{aligned}
$$

Step 3: Calculate $s_{1}=$ sum of the membership values of all undirected edges $=\sum_{a b \in E_{1}} \sigma_{1}(a b), s_{2}=$ sum of the membership values of all directed edges $=\sum_{\mathbf{a b} \in E_{2}} \sigma_{1}(\mathbf{a b})$ and $s_{3}=$ sum of the membership values of the measure of directedness of all undirected edges $=\sum_{\mathbf{a b} \in E_{2}} \tau(\mathbf{a b})$.

Step 4: Find $\max \left\{d_{\text {ifmg }}(a)\right\}$ and $\min \left\{d_{\text {ifmg }}(a)\right\}$, for all $a \in V$.

Step 5: $s_{1}=$ total undirected communication gap, $s_{2}=$ total directed communication gap, $s_{3}=$ total measure of directedness of the system.

Step 6: If $d_{i f m g}(b)=\max \left\{d_{i f m g}(a)\right\}$ and $d_{i f m g}(c)=$ $\min \left\{d_{i f m g}(a)\right\}$, then $b$ is the highest non-communicated vertex and $c$ is the lowest non-communicated vertex.

\section{Application of IFMG in social network}

In social network, communication is a system by which information are exchanged between groups or individuals. In workplace, there might be various reasons for the communication gaps (or non-communication) due to lack of time, linguistic problem, economical problem, etc. Thus, it would be beneficial for a social network to identify the total communication gap in the system. Here, we can identify or calculate the total communication gap in a social network group using the concept of IFMG.

Here we consider a communication network of five friends $F_{1}, F_{2}, F_{3}, F_{4}$ and $F_{5}$ as an IFMG $\zeta$ (see Fig. 7 ). Let $E_{1}=\left\{F_{1} F_{2}, F_{1} F_{5}, F_{2} F_{3}, F_{2} F_{4}, F_{3} F_{4}, F_{4} F_{5}\right\}$ and $E_{2}=\left\{\mathbf{F}_{1} \mathbf{F}_{3}, \mathbf{F}_{\mathbf{1}} \mathbf{F}_{\mathbf{4}}, \mathbf{F}_{\mathbf{3}} \mathbf{F}_{\mathbf{5}}, \mathbf{F}_{\mathbf{4}} \mathbf{F}_{\mathbf{3}}, \mathbf{F}_{\mathbf{5}} \mathbf{F}_{\mathbf{2}}, \mathbf{F}_{\mathbf{5}} \mathbf{F}_{1}\right\}$. Each friend represent a vertex of $\zeta$. If a friend gives full time per day in a month for communication, then the membership value of a vertex corresponding to that friend is 0 with respect to non-communication. If a friend gives no time in a month, then the membership value of the corresponding vertex is 1 with respect to non-communication. So, the membership value of a vertex lies between 0 and 1 for non-communication. For example, suppose $F_{1}$ gave an average $55 \%$ time per day in a month for communication. Then the noncommunication time for $F_{1}$ is $45 \%$ and the membership value of $F_{1}$ is 0.45 i.e., $\mu\left(F_{1}\right)=0.45$. Similarly, suppose $\mu\left(F_{2}\right)=0.55, \mu\left(F_{3}\right)=0.35, \mu\left(F_{4}\right)=0.45, \mu\left(F_{5}\right)=0.35$.

It is obvious that, the communication gap between $F_{1}$ and $F_{2}$ must be greater than or equal to minimum of noncommunication times of $F_{1}$ and $F_{2}$. Then, there exists an undirected relation $\sigma_{1}$ (say) of communication gap between $F_{1}$ and $F_{2}$ such that $\sigma_{1}\left(F_{1} F_{2}\right) \geq \min \left\{\mu\left(F_{1}\right), \mu\left(F_{2}\right)\right\}=$ $\min \{0.45,0.55\}$. We take $\sigma_{1}\left(F_{1} F_{2}\right)=0.5$. Similarly, suppose $\sigma_{1}\left(F_{1} F_{5}\right)=0.5, \sigma_{1}\left(F_{2} F_{3}\right)=0.5, \sigma_{1}\left(F_{2} F_{4}\right)=0.5$, $\sigma_{1}\left(F_{3} F_{4}\right)=0.4, \sigma_{1}\left(F_{4} F_{5}\right)=0.4$. Suppose $F_{1}$ gives the reply of all messages of $F_{3}$, but $F_{3}$ gives the reply of some (not all) messages of $F_{1}$. So there exists a directed relation $\sigma_{2}$ (say) of communication gap between $F_{1}$ and $F_{3}$ such that $\quad \sigma_{2}\left(\mathbf{F}_{\mathbf{1}} \mathbf{F}_{\mathbf{3}}\right) \geq \min \left\{\mu\left(F_{1}\right), \mu\left(F_{2}\right)\right\}=\min \{0.45,0.55\}$. We take $\sigma_{2}\left(\mathbf{F}_{\mathbf{1}} \mathbf{F}_{\mathbf{3}}\right)=0.4$. Suppose $\sigma_{2}\left(\mathbf{F}_{\mathbf{1}} \mathbf{F}_{\mathbf{4}}\right)=0.5, \sigma_{2}$ $\left(\mathbf{F}_{\mathbf{3}} \mathbf{F}_{\mathbf{5}}\right)=0.4, \sigma_{2}\left(\mathbf{F}_{\mathbf{4}} \mathbf{F}_{\mathbf{3}}\right)=0.4, \sigma_{2}\left(\mathbf{F}_{\mathbf{5}} \mathbf{F}_{\mathbf{1}}\right)=0.5, \sigma_{2} \quad\left(\mathbf{F}_{\mathbf{5}} \mathbf{F}_{\mathbf{2}}\right)$ $=0.4$. To calculate the measure of directedness of directed edges, we take $\tau\left(\mathbf{F}_{\mathbf{1}} \mathbf{F}_{\mathbf{3}}\right)=0.1, \tau\left(\mathbf{F}_{\mathbf{1}} \mathbf{F}_{\mathbf{4}}\right)=0.2, \tau\left(\mathbf{F}_{\mathbf{3}} \mathbf{F}_{\mathbf{5}}\right)=$ $0.1, \tau\left(\mathbf{F}_{\mathbf{4}} \mathbf{F}_{\mathbf{3}}\right)=0.3, \tau\left(\mathbf{F}_{\mathbf{5}} \mathbf{F}_{\mathbf{1}}\right)=0.1, \tau\left(\mathbf{F}_{\mathbf{5}} \mathbf{F}_{\mathbf{2}}\right)=0.3$.

Now, using the algorithm we have, the sum of the membership values of all undirected edges associated with the vertex $F_{1}$ is $\sigma_{1}\left(F_{1} F_{2}\right)+\sigma_{1}\left(F_{1} F_{5}\right)=0.5+0.5=1$, the sum of the membership values of all directed edges associated with the vertex $F_{1}$ towards $F_{1}$ is $\sigma_{2}\left(\mathbf{F}_{5} \mathbf{F}_{\mathbf{1}}\right)=0.4$, the sum of the membership values of all directed edges associated with the vertex $F_{1}$ from $F_{1}$ is $\sigma_{2}\left(\mathbf{F}_{\mathbf{1}} \mathbf{F}_{\mathbf{3}}\right)+$ $\sigma_{2}\left(\mathbf{F}_{\mathbf{1}} \mathbf{F}_{\mathbf{4}}\right)=0.4+0.5=0.9$.

Therefore, $d_{\text {ifmg }}\left(F_{1}\right)=1+0.4-0.9=0.5$.

Similarly, $d_{\text {ifmg }}\left(F_{2}\right)=1.4, d_{\text {ifmg }}\left(F_{3}\right)=1.3, d_{\text {ifmg }}\left(F_{4}\right)=$ $1.4, d_{\text {ifmg }}\left(F_{5}\right)=0.5$.

Thus, $\quad d_{\text {ifmg }}\left(F_{2}\right)=d_{\text {ifmg }}\left(F_{4}\right)>d_{\text {ifmg }}\left(F_{3}\right)>d_{\text {ifmg }}\left(F_{1}\right)=$ $d_{\text {ifmg }}\left(F_{5}\right)$.

Here, minimum value of the degree of all the vertices and maximum value of the degree of all the vertices

Degree of a vertex is the total contribution for communication gap of the corresponding friend in the system. Therefore according to the communication gap, the descending order of the friends are $F_{2}, F_{4}>F_{3}>F_{1}, F_{5}$.

Again,

$o(\zeta)=0.45+0.55+0.35+0.45+0.35=2.15, \quad s_{1}=$ total undirected communication gap $=$ sum of the membership values of all undirected edges $=0.5+0.5+0.5$ $+0.5+0.4+0.4=2.8, s_{2}=$ total directed communication gap=sum of the membership values of all directed edges $=0.4+0.5+0.4+0.4+0.4+0.4=2.5, \quad s_{3}=$ total measure of directedness of the system=sum of the 
membership values of the measure of directedness of all undirected edges $=0.1+0.2+0.1+0.3+0.1$ $+0.3=1.1$.

Therefore, the total undirected communication gap of the system is 2.8 , the total directed communication gap of the system is 2.5 and the measure of directedness is 1.1 .

In the above problem, the membership values of each friends (vertices) and relationship between them are uncertain. In fuzzy graph, the membership values of edges are less than or equal to the minimum of the membership values of corresponding vertices. But in this problem, the membership values of edges are not only greater than or equal to the minimum of the membership values of vertices but also there exists directed relation between vertices. This situation is absent in case of fuzzy graphs. All of these can be described using IMFG. Thus, the use of IFMG is more suitable to handle these types of problem.

The concept of inverse fuzzy graph can be used only if the undirected relationship exists between vertices. But there does not exist any directed relationship between vertices in inverse fuzzy graph. But in our problem, there may exists many directed relationships between vertices.

\section{Conclusion and future works}

Due to existence of many directed relationships between vertices in fuzzy graphs, the idea of FMGs have been introduced. Inverse relationship between vertices are applied on FMG. First, IFMG is defined with examples. Complement and isomorphic relation between two IFMGs have been analyzed. Many operations like union, intersection and join of IFMGs are initiated and theorems related to these are investigated by examples. Cartesian product of two IFMGs is defined with its properties. $\mathrm{Li}$ et al. (2020, 2021) explained Elephant herding optimization in variants, hybrids and a Survey of based on intelligent optimization. Also, Feng et al. (2021) explained Monarch butterfly optimization (MBO) algorithm, hybridizations, variants and applications in optimization techniques. Wang et al. (2019) gave comprehensive review of different $\mathrm{KH}$ algorithms. These algorithm can be helpful in our problem also. Here, an algorithm to calculate the highest and lowest communicated vertices is given in an IFMG. Then a social network group of some friends have been constructed and using the concepts of degree of vertices and size of IFMG, the decreasing order of the friends for communication gap are shown. A comparative analysis of this work with related works are also explained. The idea of inverse fuzzy relations and FMGs can be used in different types of fuzzy graphs. Some of the future works of this work are inverse bipolar fuzzy mixed graphs, mixed intuitionistic and pythagorean fuzzy graphs, inverse mixed $m$-polar fuzzy graphs, inverse interval-valued fuzzy mixed graphs, inverse interval type-2 fuzzy mixed graphs, etc.

Acknowledgements The authors would like to express their sincere gratitude to the anonymous referees for valuable suggestions, which led to great deal of improvement of the original manuscript. The first author is thankful to to meet up the financial expenditure to carry out the research work. The works described in this paper are partly supported by the Department of Higher Education, Science and Technology and Biotechnology, Government of West Bengal, India (Award No. 52-Edn (B)/5B-15/2017) and the DST-FIST, New Delhi (Sanction No. SR/FST/MS- I/2018/21).

\section{Declarations}

Conflict of interest The author declare that they have no conflict of interest.

Ethical statement This article does not contain any studies with human participants or animals performed by the author.

\section{References}

Akram M (2011) Bipolar fuzzy graphs. Inf Sci 181(24):5548-5564 Akram M (2013) Bipolar fuzzy graphs with applications. KnowlBased Syst 39:1-8

Akram M (2018) $m$-Polar fuzzy graphs: theory. Springer, Methods \& Applications, New York

Akram M, Dudek WA (2010) Interval-valued fuzzy graphs. Comput Math Appl 61(2):289-299

Akram M, Sarwar M, Dudek WA (2020) Graphs for the analysis of bipolar fuzzy information. Springer, New York

Akram M, Shahzadi G (2021) Decision-making approach based on Pythagorean dombi fuzzu soft graphs. Granul Comput 6:671-689

Akram M, Waseem N (2018) Novel applications of bipolar fuzzy graphs to decision making problems. J Appl Math Comput 56:73-91

Bhutani KR, Rosenfeld A (2003) Fuzzy end node in fuzzy graphs. Inf Sci 152:323-326

Binu M, Mathew S, Mordeson JN (2019) Connectivity index of a fuzzy graph and its application to human trafficking. Fuzzy Sets Syst 360:117-136

Binu M, Mathew S, Mordeson JN (2020) Wiener index of a fuzzy graph and application to illegal immigration networks. Fuzzy Sets Syst 384:132-147

Borozooei RA, Almallah R, Jun YB, Ghaznavi H (2020) Inverse fuzzy graphs with applications. New Math Nat Comput 16(02):397-418

Cary M (2018) Perfectly regular and perfectly edge-regular fuzzy graphs. Ann Pure Appl Math 16(2):461-469

Chen SM (1997a) Interval-valued fuzzy hypergraph and fuzzy partition. IEEE Trans Syst Man Cybern Part B Cybern 27(4):725-733

Chen SM, Hsiao WH, Jong WT (1997) Bidirectional approximate reasoning based on interval-valued fuzzy sets. Fuzzy Sets Syst 91(3):339-353

Chen SM, Hsiao WH (2000) Bidirectional approximate reasoning for rule-based systems using interval-valued fuzzy sets. Fuzzy Sets Syst 113(2):185-203

Chen SM, Yang MW, Lee LW, Yang SW (2012) Fuzzy multiple attributes group decision-making based on ranking interval type2 fuzzy sets. Expert Syst Appl 39(5):5295-5308 
Chen SM, Chang YC, Pan JS (2013) Fuzzy rules interpolation for sparse fuzzy rule-based systems based on interval type-2 Gaussian fuzzy sets and genetic algorithms. IEEE Trans Fuzzy Syst 21(3):412-425

Das K, Naseem U, Samanta S, Khan SK, De K (2021) Fuzzy mixed graphs and its application to identification of COVID19 affected central regions in India. J Intell Fuzzy Syst 40(1):1051-1064

Das K, Samanta S, De K (2021) Fuzzy chordal graphs and its properties. Int J Appl Comput Math. https://doi.org/10.1007/ s40819-021-00959-X

Feng Y, Deb S, Wang GG, Alavi AH (2021) Monarch butterfly optimization: a comprehensive review. Expert Syst Appl $168: 114418$

Gani AN, Ahamed MB (2003) Order and size in fuzzy graph. Bull Pure Appl Sci 22E(1):145-148

Ghorai G, Jacob K (2019) Recent developments on the basics of fuzzy graph theory. In: Handbook of research on advanced applications of graph theory in modern society. IGI Global. https://doi.org/10. 4018/978-1-5225-9380-5.ch018.

Ghorai G, Pal M (2016) Some isomorphic properties of $m$-polar fuzzy graphs with applications. Springerplus 5(1):2104

Knyazeva M, Belyakov S, Kacprzyk J (2018) Topological ordering on interval type-2 fuzzy graph. In: 13th international conference on theory and application of fuzzy systems and soft computing. pp 262-269

Kumar PK, Lavanya S (2017) On fuzzy diagraphs. Int J Pure Appl Math 115(3):599-606

Li J, Lei H, Alavi AH, Wang GG (2020) Elephant herding optimization: variants, hybrids, and applications. Mathematics $8(9): 1415$

Li W, Wang GG, Gandomi AH (2021) A survey of learning-based intelligent optimization algorithms. Arch Comput Methods Eng. https://doi.org/10.1007/s11831-021-09562-1

Mariappan S, Ramalingam S, Raman S, Turan GB (2019) Domination integrity and efficient fuzzy graphs. Neural Comput Appl. https://doi.org/10.1007/s00521-019-04563-5

Mathew S, Mordeson JN, Malik DS (2018) Fuzzy graph theory. Springer, New York

Mathew S, Mordeson JN, Malik DS (2018) Fuzzy graph theory with applications to human trafficking. Springer, New York

Mathew S, Sunitha MS (2009) Types of arcs in a fuzzy graph. Inf Sci 179(11):1760-1768

Mendel JM, John RI, Liu F (2006) Interval type-2 fuzzy logic systems made simple. IEEE Trans Fuzzy Syst 14(6):808-821

Mordeson JN, Peng CS (1994) Operation on fuzzy graphs. Inf Sci 79:159-170

Nirmala G, Prabavathi S (2015) Characteristics of fuzzy diagraph with generalized modus tollens using mathematical models. Int J Fuzzy Math Syst 5(1):87-97
Poulik S, Ghorai G (2020) Certain indices of graphs under bipolar fuzzy environment with applications. Soft Comput 24(7):5119-5131

Poulik S, Ghorai G (2021) Determination of journeys order based on graph's Wiener absolute index with bipolar fuzzy information. Inf Sci 545:608-619

Poulik S, Ghorai G (2020) Detour g-interior nodes and detour g-boundary nodes in bipolar fuzzy graph with applications. Hacettepe J Math Stat 49(1):106-119

Poulik S, Ghorai G (2020) Pragmatic results in Taiwan education system based IVFG \& IVNG. Soft Comput 25(2):711-724

Rashmanlou H, Muhiddin G, Sk Amanathulla, Mofidnakhaei F, Pal M (2020) A study on cubic graphs with novel application. J Intell Fuzzy Syst 40(1):1-13

Rosenfeld A (1975) Fuzzy graphs. In: Zadeh LA, Fu KS, Shimura M (eds) Fuzzy sets and their application. Academic press, New York, pp 77-95

Samanta S, Pal M (2020) Fuzzy planar graphs. IEEE Trans Fuzzy Syst 23(6):1936-1942

Samanta S, Dubey VK, Sarkar B (2021) Measure of influences in social networks. Appl Soft Comput 99:106858

Samanta S, Pal M, Mahapatra R, Das K, Bhadoria RS (2021) A study on semi-directed graphs for social media networks. Int J Comput Intell Syst 14(1):1034-1041

Singh PK (2018) Concept lattice visualization of data with $m$-polar fuzzy attribute. Granul Comput 3:123-137

Singh PK (2019) Object and attribute oriented m-polar fuzzy concept lattice using the projection operator. Granul Comput 4:545-558

Singh PK (2020) Multi-granular-based $n$-valued neutrosophic context analysis. Granul Comput 5:287-301

Turksen IB (1986) Interval valued fuzzy sets based on normal forms. Fuzzy Sets Syst 20(2):191-210

Wang GG, Gandomi AH, Alavi AH, Gong D (2019) A comprehensive review of krill herd algorithm: variants, hybrids and applications. Artif Intell Rev 51:119-148

West DB (2000) Introduction to graph theory. Pearson Education India, London

Yager RR (2020) Decision-making with measure modeled uncertain payoffs and multiple goals. Granul Comput 5:149-154

Yager RR (2021) Basic type dominance and its use in measuremodeled uncertainty in decision making. Granul Comput. https:// doi.org/10.1007/s41066-021-00271-5

Zadeh LA (1965) Fuzzy sets. Inf Control 8:338-353

Publisher's Note Springer Nature remains neutral with regard to jurisdictional claims in published maps and institutional affiliations. 